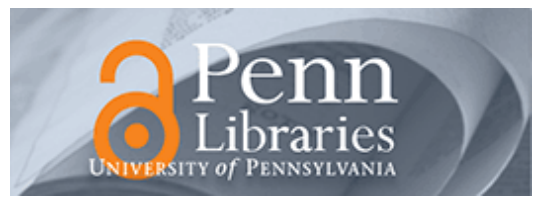

University of Pennsylvania

ScholarlyCommons

Finance Papers

Wharton Faculty Research

2009

\title{
Predictable Returns and Asset Allocation: Should a Skeptical Investor Time the Market?
}

Jessica A. Wachter

University of Pennsylvania

Missaka Warusawitharana

Follow this and additional works at: https://repository.upenn.edu/fnce_papers

Part of the Econometrics Commons, and the Finance and Financial Management Commons

\section{Recommended Citation}

Wachter, J. A., \& Warusawitharana, M. (2009). Predictable Returns and Asset Allocation: Should a Skeptical Investor Time the Market?. Journal of Econometrics, 148 (2), 162-178. http://dx.doi.org/ 10.1016/j.jeconom.2008.10.009

This paper is posted at ScholarlyCommons. https://repository.upenn.edu/fnce_papers/396

For more information, please contact repository@pobox.upenn.edu. 


\title{
Predictable Returns and Asset Allocation: Should a Skeptical Investor Time the Market?
}

\author{
Abstract \\ We investigate optimal portfolio choice for an investor who is skeptical about the degree to which excess \\ returns are predictable. Skepticism is modeled as an informative prior over the $\mathrm{R}^{2}$ of the predictive \\ regression. We find that the evidence is sufficient to convince even an investor with a highly skeptical \\ prior to vary his portfolio on the basis of the dividend-price ratio and the yield spread. The resulting \\ weights are less volatile and deliver superior out-of-sample performance as compared to the weights \\ implied by an entirely model-based or data-based view. \\ Disciplines \\ Econometrics | Finance and Financial Management
}


NBER WORKING PAPER SERIES

PREDICTABLE RETURNS AND ASSET ALLOCATION:
SHOULD A SKEPTICAL INVESTOR TIME THE MARKET?

\author{
Jessica A. Wachter \\ Missaka Warusawitharana \\ Working Paper 13165 \\ http://www.nber.org/papers/w13165
}
NATIONAL BUREAU OF ECONOMIC RESEARCH
1050 Massachusetts Avenue
Cambridge, MA 02138
June 2007

We are grateful to John Campbell, John Cochrane, Joel Dickson, Itamar Drechsler, Bjorn Eraker, Martin Lettau, Stijn Van Nieuwerburgh, Lubos Pastor, Jay Shanken, Robert Stambaugh, Alexander Stremme, Ivo Welch, Amir Yaron, Motohiro Yogo, and seminar participants at the 2005 CIRANO-CIREQ Financial Econometrics Conference, the 2006 AFA meetings, the 2006 SED meetings, the 2007 D-CAF Conference on Return Predictability, Harvard University, the Vanguard Group, and at the Wharton School for helpful comments. We are grateful for financial support from the Aronson+Johnson+Ortiz fellowship through the Rodney L. White Center for Financial Research. This manuscript does not reflect the views of the Board of Governors of the Federal Reserve or those of the National Bureau of Economic Research.

(C) 2007 by Jessica A. Wachter and Missaka Warusawitharana. All rights reserved. Short sections of text, not to exceed two paragraphs, may be quoted without explicit permission provided that full credit, including $\odot$ notice, is given to the source. 
Predictable Returns and Asset Allocation: Should a Skeptical Investor Time the Market?

Jessica A. Wachter and Missaka Warusawitharana

NBER Working Paper No. 13165

June 2007

JEL No. C11,C32,G11

\begin{abstract}
$\underline{\text { ABSTRACT }}$
Are excess returns predictable and if so, what does this mean for investors? Previous literature has tended toward two polar viewpoints: that predictability is useful only if the statistical evidence for it is incontrovertible, or that predictability should affect portfolio choice, even if the evidence is weak according to conventional measures. This paper models an intermediate view: that both data and theory are useful for decision-making. We investigate optimal portfolio choice for an investor who is skeptical about the amount of predictability in the data. Skepticism is modeled as an informative prior over the $\mathrm{R}^{\wedge} 2$ of the predictive regression. We find that the evidence is sufficient to convince even an investor with a highly skeptical prior to vary his portfolio on the basis of the dividend-price ratio and the yield spread. The resulting weights are less volatile and deliver superior out-of-sample performance as compared to the weights implied by an entirely model-based or data-based view.
\end{abstract}

Jessica A. Wachter

Department of Finance

$2300 \mathrm{SH}-\mathrm{DH}$

The Wharton School

University of Pennsylvania

3620 Locust Walk

Philadelphia, PA 19104

and NBER

jwachter@wharton.upenn.edu

Missaka Warusawitharana

Department of Research and Statistics

Board of Governors of the Federal Reserve

Mail Stop 97

20th and Constitution Ave

Washington D.C., 20551

Missaka.N.Warusawitharana@frb.gov 


\section{Introduction}

Are excess returns predictable, and if so, what does this mean for investors? In classic studies of rational valuation (e.g. Samuelson $(1965,1973)$, Shiller (1981)), risk premia are constant over time and thus excess returns are unpredictable. ${ }^{1}$ However, an extensive empirical literature has found evidence for predictability in returns on stocks and bonds by scaled-price ratios and interest rates. ${ }^{2}$

Confronted with this theory and evidence, the literature has focused on two polar viewpoints. On the one hand, if models such as Samuelson (1965) are correct, investors should maintain constant weights rather than form portfolios based on possibly spurious evidence of predictability. On the other hand, if the empirical estimates capture population values, then investors should time their allocations to a large extent, even in the presence of transaction costs and parameter uncertainty. ${ }^{3}$ Between these extremes, however, lies an interesting intermediate view: that both data and theory can be helpful in forming portfolio allocations.

This paper models this intermediate view in a Bayesian setting. We consider an investor who has a prior belief about the $R^{2}$ of the predictive regression. We implement this prior by specifying a normal distribution for the regression coefficient on the predictor variable. As the variance of this normal distribution approaches zero, the prior belief becomes dogmatic that there is no predictability. As the variance approaches infinity, the prior is diffuse: all levels of predictability are equally likely. In between, the distribution implies that the

\footnotetext{
${ }^{1}$ Examples of general-equilibrium models that imply excess returns that are largely unpredictable include Abel (1990, 1999), Backus, Gregory, and Zin (1989), Campbell (1986), Cecchetti, Lam, and Mark (1993), Kandel and Stambaugh (1991) and Mehra and Prescott (1985).

${ }^{2}$ See, for example, Fama and Schwert (1977), Keim and Stambaugh (1986), Campbell and Shiller (1988), Fama and French (1989), Cochrane (1992), Goetzmann and Jorion (1993), Hodrick (1992), Kothari and Shanken (1997), Lettau and Ludvigson (2001), Lewellen (2004), Ang and Bekaert (2006).

${ }^{3}$ See, for example, Brennan, Schwartz, and Lagnado (1997) and Campbell and Viceira (1999) for stocks and Sangvinatsos and Wachter (2005) for long-term bonds. Balduzzi and Lynch (1999) show that predictability remains important even in the presence of transaction costs, while Barberis (2000) and Xia (2001) show, respectively, that predictability remains important in the presence of estimation risk and learning. An exception is the case of buy-and-hold portfolios with horizons of many years (Barberis (2000), Cochrane (1999), Stambaugh (1999)). Brennan and Xia (2005) construct a long-run measure of expected returns and derive implications for optimal portfolios. They show that this long-run measure often implies a less extreme response to predictability than regression-based measures.
} 
investor is skeptical about predictability: predictability is possible, but it is more likely that predictability is "small" rather than "large". By conditioning this normal distribution on both the unexplained variance of returns and on the variance of the predictor variable, we create a direct mapping from the investor's prior beliefs on model parameters to a well-defined prior over the $R^{2}$.

In our empirical implementation, we consider returns on a stock index and on a long-term bond. The predictor variables are the dividend-price ratio and the yield spread between Treasuries of different maturities. We find that the evidence is sufficient to convince an investor who is quite skeptical about predictability to vary his portfolio on the basis of these variables. The resulting weights, however, are much less volatile than for an investor who allocates his portfolio purely based on data. To see whether the skeptical prior would have been helpful in the observed time series, we implement an out-of-sample analysis. We show that weights based on skeptical priors deliver superior out-of-sample performance when compared to diffuse priors, dogmatic priors, and to a simple regression-based approach.

Our study builds on previous work that has examined predictability from a Bayesian investment perspective. Kandel and Stambaugh (1996) show that predictive relations that are weak in terms of standard statistical measures can nonetheless affect portfolio choice. ${ }^{4}$ They conduct a simulation experiment such that predictability is present with modest significance, and examine the portfolio choices of a Bayesian investor who views the simulated data. In contrast, our study bases its inference on the historical time series of returns and predictor variables. We ask whether an investor whose priors imply skepticism about the existence of predictability would find it optimal to vary their investments in risky assets over time. Other studies on predictability include those of Avramov (2002) and Cremers (2002), who show that Bayesian inference can lead to superior model selection. Our paper is also related to recent work by Shanken and Tamayo (2005), who jointly model time variation in risk and expected return in a Bayesian setting. Shanken and Tamayo incorporate model-based

\footnotetext{
${ }^{4}$ Subsequently, a large literature has examined the portfolio consequences of return predictability in a Bayesian framework. Barberis (2000) considers the optimization problem of a long-horizon investor when returns are predictable. Xia (2001) considers the effect of learning about the predictive relation in a dynamic setting with hedging demands. Brandt, Goyal, Santa-Clara, and Stroud (2005) and Skoulakis (2007) extend this work to allow for uncertainty and learning about the other parameters in the predictive system. Johannes, Polson, and Stroud (2002) model the mean and volatility of returns as latent factors. In contrast to the present study, these papers assume diffuse priors.
} 
intermediate views on the relation between expected return and risk. In what follows, we compare the prior beliefs we assume to those in each of these related studies.

Besides modeling priors over the $R^{2}$, our study also incorporates the findings of Stambaugh (1999). Stambaugh shows that incorporating the first observation on the predictor variable into the likelihood can make a substantial difference for portfolio choice; previous studies had conditioned on this observation. Moreover, the choice among "uninformative" priors can make a difference as well: a prior that is uninformative in the sense of Jeffreys (1961) has different properties than the uninformative priors that have been chosen previously in the portfolio choice literature. Building on the work of Stambaugh, this study also incorporates information contained in the first observation on the predictor variable, and makes use of the limiting Jeffreys priors of Zellner (1996). We show that Jeffreys invariance theory offers an independent justification for defining the prior over the change in the $R^{2}$. As the degree of skepticism goes to zero, the prior satisfies the Jeffreys condition for invariance.

Our use of model-based informative priors has parallels in a literature that examines the portfolio implications of the cross-section of stock returns. Motivated by the extreme weights and poor out-of-sample performance of mean-variance efficient portfolios (Best and Grauer (1991), Green and Hollifield (1992)), Black and Litterman (1992) propose using market weights as a benchmark, in effect using both data and the capital asset pricing model to form portfolios. Recently, Bayesian studies such as Pastor (2000), Avramov (2004) and Wang (2005) construct portfolios incorporating informative beliefs about cross-sectional asset pricing models. ${ }^{5}$ Like the present study, these studies show that allowing models to influence portfolio selection can be superior to using the data alone. While these studies focus on the cross-section of returns, we apply these ideas to the time series.

The remainder of this paper is organized as follows. Section 1 describes the assumptions on the likelihood and prior, the calculation of the posterior, and the optimization problem of the investor. Section 2 applies these results to data on stock and bond returns, describes the

\footnotetext{
${ }^{5}$ Related approaches to improving performance of efficient portfolios include Bayesian shrinkage (Jobson and Korkie (1980), Jorion (1985)) and portfolio constraints (Frost and Savarino (1988), Jagannathan and Ma (2003)). Cvitanic, Lazrak, Martellini, and Zapatero (2006) incorporate analyst forecasts in a dynamic setting with parameter uncertainty and learning. Garlappi, Uppal, and Wang (2007) take a multi-prior approach to portfolio allocation that allows for ambiguity aversion. Tu and Zhou (2007) impose priors that ensure that portfolio weights fall into a certain range. Unlike the present study, these papers assume that the true distribution of returns is iid and focus on the cross-section.
} 
posterior distributions, the portfolio weights, and the out-of-sample performance across different choices of priors. These sections assume, for simplicity, that there is a single predictor variable. Section 3 extends the methods to allow for multiple predictor variables. Section 4 concludes.

\section{Portfolio choice for a skeptical investor}

Given observations on returns and a predictor variable, how should an investor allocate his wealth? One approach would be to estimate the predictability relation, treat the point estimates as known, and solve for the portfolio that maximizes utility. An alternative approach, adopted in Bayesian studies, is to specify prior beliefs on the parameters. The prior represents the investor's beliefs about the parameters before viewing data. After viewing data, the prior is updated to form a posterior distribution; the parameters are then integrated out to form a predictive distribution for returns, and utility is maximized with respect to this distribution. This approach incorporates the uncertainty inherent in estimation into the decision problem (see Klein and Bawa (1976), Bawa, Brown, and Klein (1979), Brown (1979)). ${ }^{6}$ Rather than assuming that the investor knows the parameters, it assumes, realistically, that the investor estimates the parameters from the data. Moreover, this approach allows for prior information, perhaps motivated by economic models, to enter into the decision process. This section describes the specifics of the likelihood function, the prior, and the posterior used in this study.

\section{$1.1 \quad$ Likelihood}

This subsection constructs the likelihood function. Let $r_{t+1}$ denote an $N \times 1$ vector of returns on risky assets in excess of a riskless asset from time $t$ to $t+1$, and $x_{t}$ a scalar predictor variable at time $t$. The investor observes data on returns $r_{1}, \ldots, r_{T}$, and the predictor variable $x_{0}, \ldots, x_{T}$. Let

$$
D \equiv\left\{r_{1}, \ldots, r_{T}, x_{0}, x_{1}, \ldots, x_{T}\right\}
$$

\footnotetext{
${ }^{6}$ Like these and the portfolio choice papers cited in the introduction, this paper studies an investor who should not be viewed as representative. By definition, the representative investor must hold the market portfolio.
} 
represent the total data available to the investor. Our initial assumption is that there is a single predictor variable that has the potential to predict returns on (possibly) multiple assets. Allowing multiple predictor variables complicates the problem without contributing to the intuition. For this reason, we postpone the discussion of multiple predictor variables until Section 3.

The data generating process is assumed to be

$$
\begin{aligned}
& r_{t+1}=\alpha+\beta x_{t}+u_{t+1} \\
& x_{t+1}=\theta_{0}+\theta_{1} x_{t}+v_{t+1},
\end{aligned}
$$

where

$$
\left[\begin{array}{c}
u_{t+1} \\
v_{t+1}
\end{array}\right] \mid r_{t}, \ldots, r_{1}, x_{t}, \ldots, x_{0} \sim N(0, \Sigma),
$$

$\alpha$ and $\beta$ are $N \times 1$ vectors and $\Sigma$ is an $(N+1) \times(N+1)$ symmetric and positive definite matrix. It is useful to partition $\Sigma$ so that

$$
\Sigma=\left[\begin{array}{cc}
\Sigma_{u} & \Sigma_{u v} \\
\Sigma_{v u} & \Sigma_{v}
\end{array}\right]
$$

where $\Sigma_{u}$ is the variance-covariance matrix of $u_{t+1}, \sigma_{v}^{2}=\Sigma_{v}$ is the variance of $v_{t+1}, \Sigma_{u v}$ is the $N \times 1$ vector of covariances of $v_{t+1}$ with each element of $u_{t+1}$, and $\Sigma_{v u}=\Sigma_{u v}^{\top}$. This likelihood is a multi-asset analogue of that assumed by Kandel and Stambaugh (1996), Campbell and Viceira (1999), and many subsequent studies.

It is helpful to group the regression parameters in (1) and (2) into a matrix:

$$
B=\left[\begin{array}{ll}
\alpha^{\top} & \theta_{0} \\
\beta^{\top} & \theta_{1}
\end{array}\right]
$$

and to define matrices of the observations on the the left hand side and right hand side variables:

$$
Y=\left[\begin{array}{cc}
r_{1}^{\top} & x_{1} \\
\vdots & \vdots \\
r_{T}^{\top} & x_{T}
\end{array}\right], \quad X=\left[\begin{array}{cc}
1 & x_{0} \\
\vdots & \vdots \\
1 & x_{T-1}
\end{array}\right]
$$

As shown in Barberis (2000) and Kandel and Stambaugh (1996), the likelihood conditional on the first observation takes the same form as in a regression model with non-stochastic 
regressors. Let $p\left(D \mid B, \Sigma, x_{0}\right)$ denote the likelihood function. From results in Zellner (1996), it follows that

$$
p\left(D \mid B, \Sigma, x_{0}\right)=|2 \pi \Sigma|^{-\frac{T}{2}} \exp \left\{-\frac{1}{2} \operatorname{tr}\left[(Y-X B)^{\top}(Y-X B) \Sigma^{-1}\right]\right\},
$$

where $\operatorname{tr}(\cdot)$ denotes the sum of the diagonal elements of a matrix. ${ }^{7}$

The likelihood function (4) conditions on the first observation of the predictor variable, $x_{0}$. Stambaugh (1999) argues for treating $x_{0}$ and $x_{1}, \ldots, x_{T}$ symmetrically: as random draws from the data generating process. If the process for $x_{t}$ is stationary and has run for a substantial period of time, then results in Hamilton (1994, p. 53) imply that $x_{0}$ is a draw from a normal distribution with mean

$$
\mu_{x} \equiv E\left[x_{t} \mid B, \Sigma\right]=\frac{\theta_{0}}{1-\theta_{1}}
$$

and variance

$$
\sigma_{x}^{2} \equiv E\left[\left(x_{t}-\mu_{x}\right)^{2} \mid B, \Sigma\right]=\frac{\sigma_{v}^{2}}{1-\theta_{1}^{2}} .
$$

Combining the likelihood of the first observation with the likelihood of the remaining $T$ observations produces

$$
\begin{aligned}
p(D \mid B, \Sigma)= & p\left(D \mid x_{0}, B, \Sigma\right) p\left(x_{0} \mid B, \Sigma\right) \\
= & \left(2 \pi \sigma_{x}^{2}\right)^{-\frac{1}{2}}|2 \pi \Sigma|^{-\frac{T}{2}} \\
& \quad \times \exp \left\{-\frac{1}{2} \sigma_{x}^{-2}\left(x_{0}-\mu_{x}\right)^{2}-\frac{1}{2} \operatorname{tr}\left[(Y-X B)^{\top}(Y-X B) \Sigma^{-1}\right]\right\} .
\end{aligned}
$$

Equation (7) is the likelihood function used in our analysis. Following Box, Jenkins, and Reinsel (1970), we refer to (7) as the exact likelihood, and to (4) as the conditional likelihood.

\subsection{Prior beliefs}

This subsection describes the prior. We specify a class of prior distributions that range from being "uninformative" in a sense we will make precise, to "dogmatic". The uninformative

\footnotetext{
${ }^{7}$ Maximizing the conditional likelihood function (4) is equivalent to running a vector auto-regression. Resulting estimates for $\beta$ are biased (see Bekaert, Hodrick, and Marshall (1997), Nelson and Kim (1993) and Stambaugh (1999)), and standard asymptotics provide a poor approximation to the distribution of test statistics in small samples (Cavanagh, Elliott, and Stock (1995), Elliott and Stock (1994), Mankiw and Shapiro (1986), Richardson and Stock (1989)). An active literature based in classical statistics focuses on correcting for these problems (e.g. Amihud and Hurvich (2004), Campbell and Yogo (2006), Eliasz (2004), Ferson, Sarkissian, and Simin (2003), Lewellen (2004), Torous, Valkanov, and Yan (2004)).
} 
priors imply that all amounts of predictability are equally likely, while the dogmatic priors rule out predictability all together; the investor believes that returns are not predictable regardless of what data are observed. Between these extremes lie priors that downweight the return predictability. These informative priors imply that large values of the $R^{2}$ from predictive regressions are unlikely, but not impossible.

The most obvious parameter that determines the degree of predictability is $\beta$. Set $\beta$ to zero, and there is no predictability in the model. However, it is difficult to think of prior beliefs about $\beta$ in isolation from beliefs about other parameters. For example, a high variance of $x_{t}$ might lower one's prior on $\beta$, while a large residual variance of $r_{t}$ might raise it. Rather than placing a prior on $\beta$ directly, we instead place a prior on "normalized" $\beta$, that is $\beta$ adjusted for the variance of $x$ and the variance of $u$. Let $C_{u}$ be the Cholesky decomposition of $\Sigma_{u}$, i.e. $C_{u} C_{u}^{\top}=\Sigma_{u}$. Then

$$
\eta=C_{u}^{-1} \sigma_{x} \beta
$$

is normalized $\beta$. We assume that prior beliefs on $\eta$ are given by

$$
\eta \sim N\left(0, \sigma_{\eta}^{2} I_{N}\right)
$$

where $I_{N}$ is the $N \times N$ identity matrix. ${ }^{8}$ We implement these prior beliefs by specifying a hierarchical prior. The prior for $\beta$ is conditional on the remaining parameters:

$$
p(B, \Sigma)=p\left(\beta \mid \alpha, \theta_{0}, \theta_{1}, \Sigma\right) p\left(\alpha, \theta_{0}, \theta_{1}, \Sigma\right) .
$$

Then (8) implies

$$
\beta \mid \alpha, \theta_{0}, \theta_{1}, \Sigma \sim N\left(0, \sigma_{\eta}^{2} \sigma_{x}^{-2} \Sigma_{u}\right)
$$

Because $\sigma_{x}$ is a function of $\theta_{1}$ and $\sigma_{v}$, the prior on $\beta$ is also implicitly a function of these parameters. The parameter $\sigma_{\eta}$ indexes the degree to which the prior is informative. We show that as $\sigma_{\eta} \rightarrow \infty$, the prior over $\beta$ becomes uninformative; all values of $\beta$ are viewed as equally likely. As $\sigma_{\eta} \rightarrow 0$, the prior converges to a point mass at zero and the prior beliefs assign a probability of 1 to no predictability.

Finite positive values of $\sigma_{\eta}$ involve some skepticism about the amount of predictability in the data. The dependence between $\beta, \sigma_{x}$, and $\Sigma_{u}$ allow this skepticism to be expressed in

\footnotetext{
${ }^{8}$ Formally, we can consider a prior on the parameters $p\left(\alpha, \eta, \theta_{0}, \theta_{1}, \Sigma\right)=p(\eta) p\left(\alpha, \theta_{0}, \theta_{1}, \Sigma\right)$, where $p(\eta)$ is defined by (8).
} 
terms of the $R^{2}$ from the regressions (1). This is easiest to see in the case of a single risky asset. When there is a single risky asset, the population $R^{2}$ is equal to

$$
R^{2}=\beta^{2} \sigma_{x}^{2}\left(\beta^{2} \sigma_{x}^{2}+\Sigma_{u}\right)^{-1}=\frac{\eta^{2}}{\eta^{2}+1}
$$

where $\Sigma_{u}$ is now a scalar. Therefore a prior over $\eta$ translates into a prior over the $R^{2}$. When there are $N$ risky assets, the distribution on $\eta$ implies a distribution for the $R^{2}$ of each asset. Moreover, it implies a distribution for the maximum $R^{2}$ achievable on a portfolio of assets. Let $w$ be an $N \times 1$ vector of asset weights. Then

$$
\begin{aligned}
\max _{w} R^{2} & =\max _{w} \frac{w^{\top} \beta \beta^{\top} \sigma_{x}^{2}}{w^{\top} \beta \beta^{\top} w \sigma_{x}^{2}+w^{\top} \Sigma_{u} w} \\
& =\frac{\eta^{\top} \eta}{\eta^{\top} \eta+1}
\end{aligned}
$$

where the second line is shown in Appendix A. ${ }^{9}$

By formulating the prior for $\beta$ in terms of $\sigma_{x}$ and $\Sigma_{u}$, we put a prior on the $R^{2}$ of the predictability equation. For this to work, it is necessary to condition on both $\sigma_{x}$ and $\Sigma_{u}$. Consider, for example, two different state variables, one with a lower unconditional volatility $\left(\sigma_{x}\right)$ than the other. Our prior implies that the state variable with the lower unconditional volatility puts more weight on large values of $\beta$, all else equal. ${ }^{10}$ The reason is that a lower $\sigma_{x}$ implies a lower $R^{2}$ for any given level of $\beta$ : an investor who is skeptical about predictability would therefore be willing to consider larger values of $\beta$ in the case of lower $\sigma_{x}$ because these would still be consistent with a low $R^{2}$. In the limit as $\sigma_{x}$ approaches zero, the prior on $\beta$ flattens because even an arbitrarily large $\beta$ implies that returns are almost unpredictable.

Conditioning on $\Sigma_{u}$ is also important. Consider for simplicity the case of a single risky asset. If $\Sigma_{u}$ is large, then even large values of $\beta$ relative to $\sigma_{x}$ translate into low amounts of predictability because the "signal" $\beta x_{t}$ is overwhelmed by the noise $u_{t+1}$. Large values of

\footnotetext{
${ }^{9}$ This prior distribution could easily be modified to impose other restrictions on the coefficients $\beta$. In the context of predicting equity returns, Campbell and Thompson (2007) suggest disregarding estimates of $\beta$ if the expected excess return is negative, or if $\beta$ has an opposite sign to that suggested by theory. In our model, these restrictions could be imposed by assigning zero prior weight to the appropriate regions of the parameter space. One could also consider a non-zero mean for $\beta$, corresponding to a prior belief that favors predictability of a particular sign. For simplicity, we focus on priors that apply to any predictor variable on possibly multiple assets, and leave these extensions to future work.

${ }^{10} \mathrm{By}$ "large" values of $\beta$, in this and the next paragraph we mean vectors $\beta$ whose elements are large in absolute value.
} 
$\beta$ are still consistent with low values of the $R^{2}$ when $u_{t+1}$ is large. In the case of multiple risky assets, conditioning on $\Sigma_{u}$ plays an additional role: it implies that the investor does not downweight predictability on specific assets per se, but on predictability on the system of assets. It is not possible to obtain a high $R^{2}$ by cleverly combining assets into a portfolio.

For the remaining parameters, we choose a prior that is uninformative in the sense of Jeffreys (1961). Jeffreys argues that a reasonable property of a "no-information" prior is that inference be invariant to one-to-one transformations of the parameter space. Given a set of parameters $\mu$, data $D$, and a $\log$-likelihood $l(\mu ; D)$, Jeffreys shows that invariance is equivalent to specifying a prior as

$$
p(\mu) \propto\left|-E\left(\frac{\partial^{2} l}{\partial \mu \partial \mu^{\top}}\right)\right|^{1 / 2} .
$$

Besides invariance, this formulation of the prior has other advantages such as minimizing asymptotic bias and generating confidence sets that are similar to their classical counterparts (see Phillips (1991)). ${ }^{11}$

We follow the approach of Stambaugh (1999) and Zellner (1996), and derive a limiting Jeffreys prior as explained in Appendix C. This limiting prior is given by taking an unconditional expectation in (12) and takes the form

$$
p\left(\alpha, \theta_{0}, \theta_{1}, \Sigma\right) \propto \sigma_{x}\left|\Sigma_{u}\right|^{1 / 2}|\Sigma|^{-\frac{N+4}{2}},
$$

for $\theta_{1} \in(-1,1)$, and zero otherwise. Therefore the joint prior is given by

$$
\begin{aligned}
p(B, \Sigma) & =p\left(\beta \mid \alpha, \theta_{0}, \theta_{1}, \Sigma\right) p\left(\alpha, \theta_{0}, \theta_{1}, \Sigma\right) \\
& \propto \sigma_{x}^{N+1}|\Sigma|^{-\frac{N+4}{2}} \exp \left\{-\frac{1}{2} \beta^{\top}\left(\sigma_{\eta}^{2} \sigma_{x}^{-2} \Sigma_{u}\right)^{-1} \beta\right\}
\end{aligned}
$$

\footnotetext{
${ }^{11}$ The notion of an uninformative prior in a time-series setting is a matter of debate. One approach is to ignore the time-series aspect of (1) and (2), treating the right hand side variable as exogenous. This implies a flat prior for $\alpha, \beta, \theta_{0}$, and $\theta_{1}$. When applied in a setting with exogenous regressors, this approach leads to Bayesian inference which is quite similar to classical inference (Zellner (1996)). However, Sims and Uhlig (1991) show that applying the resulting priors in a time series setting leads to different inference than classical procedures when $x_{t}$ is highly persistent. Phillips (1991) derives an exact Jeffreys (1961) prior and shows that the inference with this prior leads to different conclusions than inference with a prior that is flat for the regression coefficients. As a full investigation of these issues is outside the scope of this study, we focus on the Jeffreys prior. Replacing the prior in (13) with one that is implied by exogenous regressors gives results that are similar to our current ones; these are available from the authors.
} 
(note that $\sigma_{\eta}$ is a constant). Because Jeffreys priors involve the likelihood function, typically they require "prior" knowledge of the data. An advantage of the limiting Jeffreys prior is that it does not require this knowledge. We emphasize that, in (13) and (14), $\sigma_{x}$ is a nonlinear function of the autoregressive coefficient $\theta_{1}$ and volatility of the shock to the predictor variable $\sigma_{v}$.

Jeffreys invariance theory provides an independent justification for modeling priors on $\beta$ as in (9). Appendix B shows that the limiting Jeffreys prior for $B$ and $\Sigma$ equals

$$
p(B, \Sigma) \propto \sigma_{x}^{N+1}|\Sigma|^{-\frac{N+4}{2}}
$$

This prior corresponds to (14) as $\sigma_{\eta}$ approaches infinity. Modeling the prior for $\beta$ as depending on $\sigma_{x}$ not only has an interpretation in terms of the $R^{2}$, but also implies that an infinite prior variance represents ignorance as defined by Jeffreys (1961). Note that a prior on $\beta$ that is independent of $\sigma_{x}$ would not have this property. Because the priors in (14) combine an informative ("skeptical") prior on $\beta$ with a Jeffreys prior on the remaining parameters, we refer to these as skeptical Jeffreys priors.

Figure 1 depicts the distribution of the $R^{2}$ implicit in our prior beliefs. The figure shows the probability that the $R^{2}$ exceeds some value $k, P\left(R^{2}>k\right)$, as a function of $k$; it is therefore one minus the cumulative distribution function for the $R^{2}$. We construct this figure by simulating draws of $\eta$ from (8) and, for each draw, constructing a draw from the $R^{2}$ distribution using (10). Note that for $\sigma_{\eta}=0$, the investor assigns zero probability to a positive $R^{2}$; for this reason $P\left(R^{2}>k\right)$ is equal to one at zero and is zero elsewhere. As $\sigma_{\eta}$ increases, the investor assigns non-zero probability to positive values of the $R^{2}$. For $\sigma_{\eta}=.04$, the probability that the $R^{2}$ exceeds .02 is .0005 . For $\sigma_{\eta}=.08$, the probability that the $R^{2}$ exceeds .02 is .075. Finally when $\sigma_{\eta}$ is large, approximately equal probabilities are assigned to all values of the $R^{2}$. This is the diffuse prior that assigns no skepticism to the data. In what follows, we will consider the implications of these four priors for the individual's investment decisions. We will refer to them by using the corresponding probabilities that the $R^{2}$ exceeds .02. We note, however, that we focus on .02 for convenience; any number between 0 and 1 could be substituted. 


\section{Comparison with related studies}

In this section we have described one way of modeling prior information. We now compare this approach to that used in other return predictability studies that make use of informative priors. These include Kandel and Stambaugh (1996), Avramov (2002, 2004), Cremers (2002) and Shanken and Tamayo (2005). ${ }^{12}$ With the exception of Shanken and Tamayo (2005), these studies do not focus on informative priors over return predictability. Nonetheless, they do make use of informative priors, so it is instructive to compare their approaches to the approach that we take here.

Kandel and Stambaugh (1996) derive posteriors assuming the investor has seen, in addition to the actual data, a "prior" sample of the data that has moments equal to those of the actual sample except but without predictability. ${ }^{13}$ Avramov $(2002,2004)$ also takes this approach. As this approach serves to reduce posterior estimates relative to sample estimates, it has similar effects to introducing an informative prior as we do here. We do not to take this approach because constructing this prior sample requires knowledge of moments of the data for the actual sample. It is therefore difficult to justify this procedure in decision-theoretic terms, as it assumes that the investor knows moments of the future time series of the data.

Cremers (2002) specifies informative prior beliefs about the time series that highlight the importance of the expected $R^{2}$ in the predictability equation. Cremers's priors assume knowledge of sample moments of the predictor variable. In a setting where regressors were exogenous this might not present a problem. However, when the regressor is stochastic and correlated with returns, it is necessary to assume that the investor knows the sample moments without having seen the predictor variable. This also is difficult to justify in decision-theoretic terms. ${ }^{14}$ An advantage of our approach over these previous studies is that

\footnotetext{
${ }^{12}$ Goyal and Welch (2004) present an "encompassing forecast", which, while not Bayesian, has similar implications in that it downweights the predictability coefficient estimated from the data. Bayesian methods can be seen as formalizing this approach.

${ }^{13}$ Kandel and Stambaugh discuss the appeal of holding the distribution of the $R^{2}$ constant, and for this reason, set the length of the prior sample to increase in the number of predictor variables.

${ }^{14}$ Data-based procedures for forming priors are often referred to as "empirical Bayes". However, at least in its classic applications, empirical Bayes implies either the the use of data that is known prior to the decision problem at hand or data from the population from which the parameter of interest can be drawn (Robbins (1964), Berger (1985)). For example, if one is forming a prior on a expected return for a particular security, one might use the average expected return of securities for that industry (Pastor and Stambaugh (1999)).
} 
our priors are parsimonious but do not require knowledge of future data. ${ }^{15}$

Another approach is adopted by Shanken and Tamayo (2005). Shanken and Tamayo model time-variation in risk as well as in expected returns. Like our priors, the priors in Shanken and Tamayo represent a model-based view that is intermediate between complete faith in a model and complete faith in the data. However, their formulation of priors is less parsimonious, requiring ten parameters in the case of a single asset (a broad stock market portfolio) and predictor variable (the dividend-yield). The prior values are specific to these variables and do not transfer easily to other assets or new predictor variables. The advantage of our method is that it expresses the informativeness of the agent's prior beliefs as a single number which can be mapped into beliefs about the maximum $R^{2}$. This is the case regardless of the number of risky assets or the number and characteristics of the predictor variables.

Our priors are in fact reminiscent of the choice of prior on the intercepts in cross-sectional studies. Pastor and Stambaugh (1999) and Pastor (2000) place an informative prior on the vector of intercepts from regressions of returns on factors in the cross-section. Building on ideas of MacKinlay (1995), these studies argue that failure to condition the intercepts on the residual variance could lead to very high Sharpe ratios, because there would be nothing to prevent a low residual variance draw from occurring simultaneously with a high intercept draw. Bayesian portfolio choice studies (Baks, Metrick, and Wachter (2001), Jones and Shanken (2005), Pastor and Stambaugh (2002)) place an informative prior on estimates of mutual fund skill (intercepts from regressions of returns on factors), and argue based on related ideas that this informative prior should be conditioned on the residual variance of the fund. In the present study, $\beta$ plays a role that is roughly analogous to the intercept in these previous studies. $\beta=0$ implies no predictability, and hence no "mispricing". As in these previous studies, conditioning $\beta$ on volatility measures ensures that a high draw of $\beta$ could not coincide with a low draw of $\Sigma_{u}$. However, in the time-series setting, it is not sufficient to condition $\beta$ on $\Sigma_{u} ; \beta$ must also be conditioned on $\sigma_{x}$ in order to produce a well-defined distribution for quantities of interest.

\footnotetext{
${ }^{15}$ Generally Jeffreys priors do require knowledge of future data, because they involve taking derivatives of the likelihood function. Our limiting Jeffreys priors, however, integrate out over the data and are therefore not subject to this critique.
} 


\subsection{Posterior}

This section shows how the likelihood of Section 1.1 and the prior of Section 1.2 combine to form the posterior distribution. From Bayes' rule, it follows that the joint posterior for $B, \Sigma$ is given by

$$
p(B, \Sigma \mid D) \propto p(D \mid B, \Sigma) p(B, \Sigma),
$$

where $p(D \mid B, \Sigma)$ is the likelihood and $p(B, \Sigma)$ is the prior. Substituting in the prior (14) and the likelihood (7) produces

$$
\begin{array}{r}
p(B, \Sigma \mid D) \propto \sigma_{x}^{N}|\Sigma|^{-\frac{T+N+4}{2}} \exp \left\{-\frac{1}{2} \beta^{\top}\left(\sigma_{\eta}^{2} \sigma_{x}^{-2} \Sigma_{u}\right)^{-2} \beta\right\} \exp \left\{-\frac{1}{2} \sigma_{x}^{-2}\left(x_{0}-\mu_{x}\right)^{2}\right\} \\
\exp \left\{-\frac{1}{2} \operatorname{tr}\left[(Y-X B)^{\top}(Y-X B) \Sigma^{-1}\right]\right\}
\end{array}
$$

as a posterior.

This posterior does not take the form of a standard density function because of the presence of $\sigma_{x}^{2}$ in the prior and in the term in the likelihood involving $x_{0}$ (note that $\sigma_{x}^{2}$ is a nonlinear function of $\theta_{1}$ and $\sigma_{v}$ ). However, we can sample from the posterior using the Metropolis-Hastings algorithm (see Chib and Greenberg (1995)). Define column vectors

$$
\begin{aligned}
b & =\operatorname{vec}(B)=\left[\alpha_{1}, \beta_{1}, \cdots \alpha_{N}, \beta_{N}, \theta_{0}, \theta_{1}\right]^{\top} \\
b_{1} & =\left[\alpha_{1}, \beta_{1}, \cdots \alpha_{N}, \beta_{N}\right]^{\top} \\
b_{2} & =\left[\theta_{0}, \theta_{1}\right]^{\top} .
\end{aligned}
$$

The Metropolis-Hastings algorithm is implemented "block-at-a-time", by first sampling from $p(\Sigma \mid b, D)$, then $p\left(b_{1} \mid b_{2}, \Sigma, D\right)$, and finally $p\left(b_{2} \mid b_{1}, \Sigma, D\right)$. The proposal density for the conditional probability of $\Sigma$ is the inverted Wishart with $T+2$ degrees of freedom and scale factor of $(Y-X B)^{\top}(Y-X B)$. The accept-reject algorithm of Chib and Greenberg (1995, Section 5) is used to sample from the target density, which takes the same form as (16). The proposal densities for $b_{1}$ and $b_{2}$ are multivariate normal. For $b_{1}$, the proposal and the target are equivalent, while for $b_{2}$, the accept-reject algorithm is used to sample from the target density. Details are given in Appendix D. As described in Chib and Greenberg, drawing successively from the conditional posteriors for $\Sigma, b_{1}$, and $b_{2}$ produces a density that converges to the full posterior. 


\subsection{Predictive distribution and portfolio choice}

This section describes how we determine optimal portfolio choice based on the posterior distribution. Consider an investor who maximizes expected utility at time $T+1$ conditional on information available at time $T$. The investor solves

$$
\max E_{T}\left[U\left(W_{T+1}\right) \mid D\right]
$$

where $W_{T+1}=W_{T}\left[w_{T}^{\top} r_{T+1}+r_{f, T}\right], w_{T}$ are the weights in the $N$ risky assets, and $r_{f, T}$ is the total return on the riskless asset from time $T$ to $T+1$ (recall that $r_{T+1}$ is a vector of excess returns). The expectation in (17) is taken with respect to the predictive distribution

$$
p\left(r_{T+1} \mid D\right)=\int p\left(r_{T+1} \mid x_{T}, B, \Sigma\right) p(B, \Sigma \mid D) d B d \Sigma
$$

Following previous single-period portfolio choice studies (see, e.g. Baks, Metrick, and Wachter (2001) and Pastor (2000)), we assume that the investor has quadratic utility. The advantage of quadratic utility is that it implies a straightforward mapping between the moments of the predictive distribution of returns and portfolio choice. However, because our method produces an entire distribution function for returns, it can be applied to other utility functions, and to buy-and-hold investors with horizons longer than one quarter.

Let $\tilde{E}$ denote the expectation and $\tilde{V}$ the variance-covariance matrix of the $N$ assets corresponding to the predictive distribution (18). For a quadratic-utility investor, optimal weights $w^{*}$ in the $N$ assets are given by

$$
w^{*}=\frac{1}{A} \tilde{V}^{-1} \tilde{E}
$$

where $A$ is a parameter determining the investor's risk aversion. The weight in the riskless bond is equal to $1-\sum_{i=1}^{N} w_{i}^{*}$. Note that our method makes no assumptions on the riskfree rate as the level of the riskfree rate is not relevant for portfolio choice under our assumptions.

Given draws from the posterior distribution of the parameters $\alpha^{j}, \beta^{j}, \Sigma_{u}^{j}$, and a value of $x_{t}$, a draw from the predictive distribution of asset returns is given by

$$
r^{j}=\alpha^{j}+\beta^{j} x_{t}+u^{j}
$$

where $u^{j} \sim N\left(0, \Sigma_{u}^{j}\right)$. The optimal portfolio is then the solution to (19), with the mean and variance computed by simulating draws $r^{j}$. 


\section{Results}

We consider the problem of a quadratic utility investor who allocates wealth between a riskless asset, a long-term bond, and a stock index. We estimate two versions of the system given in (1)-(3), one with the dividend-price ratio as the predictor variable and one with the yield spread. An appeal of these variables is that they are related to excess returns through present value identities for bonds and stocks (see Campbell and Shiller $(1988,1991)$ ).

\subsection{Data}

All data are obtained from the Center for Research on Security Prices (CRSP). Excess stock and bond returns are formed by subtracting the quarterly return on the three-month Treasury bond from the quarterly return on the value-weighted NYSE-AMEX-NASDAQ index and the ten-year Treasury bond (from the CRSP indices file) respectively. The dividend-price ratio is constructed from monthly return data on the stock index as the sum of the previous twelve months of dividends divided by the current price. The natural logarithm of the dividendprice ratio is used as the predictor variable. The yield spread is equal to the continuously compounded yield on the zero-coupon five year bond (from the Fama-Bliss data set) less the continuously compounded yield on the three-month bond. Data on bond yields are available from the second quarter of 1952. We therefore consider quarterly observations from the second quarter of 1952 until the last quarter of 2004.

\subsection{Posterior means, expected returns and portfolios conditional on the full sample}

This section quantitatively describes the posterior beliefs of an investor who views the entire

data set. For both predictor variables, one million draws from the posterior distribution are simulated as described in Section 1.3. An initial 100,000 "burn-in" draws are discarded.

We first examine the posterior distribution over the maximum $R^{2}$. Because our empirical implementation assumes two assets, we compute the prior distribution of (11) assuming $N=2$. The left panel of Figure 2 reports the probability that the maximum $R^{2}$ exceeds $k$, as a function of $k$ for both the prior with $\sigma_{\eta}=.08$ and for the posterior distribution implied by this prior when the dividend-price ratio is the predictor variable. Below $k=.02$, the 
posterior probability that the $R^{2}$ exceeds $k$ is above the prior probability. Above .02 , the posterior probability that the $R^{2}$ exceeds $k$ is lower for the posterior than for the prior. The right panel of Figure 2 shows the probability density function of the posterior and of the prior. While the prior density is decreasing in the $R^{2}$ over this range, the posterior density is hump-shaped with a maximum at about 0.02 .

Figure 3 shows analogous results for the yield spread. In this case, the agent places more weight on relatively high values of the $R^{2}$ as compared with results for the dividend yield. The probability that the $R^{2}$ exceeds $k$ is larger for the posterior than the prior across the entire range that we consider. The posterior distribution for the $R^{2}$ still peaks at about 0.02 , but falls off less quickly than in the case of the dividend yield.

Table 1 reports posterior means for values of $\sigma_{\eta}$ equal to $0, .04, .08$, and $\infty$. To emphasize the economic significance of these priors, we report the corresponding probabilities that the $R^{2}$ exceeds .02: 0, .0005, .075, and .999. ${ }^{16}$ The predictor variable is the dividend-price ratio. Posterior standard deviations are reported in parentheses. The table also shows results from estimation by ordinary least squares (OLS). For the OLS values, standard errors are reported in parentheses.

As Table 1 shows, the dividend-price ratio predicts stock returns but not bond returns. The posterior mean for the $\beta$ for bond returns is negative and small in magnitude. The posterior mean for the $\beta$ for stock returns is positive for all of the priors we consider and for the OLS estimate. For the diffuse prior, the posterior mean of $\beta$ equals to 1.46, below the OLS estimate of 2.72. As the prior becomes more informative, the posterior mean for $\beta$ becomes smaller: for $P\left(R^{2}>.02\right)=.075$, the estimate is 1.41 , while for $P\left(R^{2}>.02\right)=.0005$, it is 0.69 .

Even though all of the priors are uninformative with respect to the autoregressive coefficient $\theta_{1}$, the posterior mean of $\theta_{1}$ nonetheless increases as the priors become more informative over $\beta$. The reason is the negative correlation between draws for $\theta_{1}$ and draws for $\beta$. As Stambaugh (1999) shows, the negative correlation between shocks to returns and shocks to the predictor variable implies that when $\beta$ is below its OLS value, $\theta_{1}$ tends to be above its OLS value. The reason is that if $\beta$ is below its OLS value, it must be that the lagged predictor variable and returns have an unusually high covariance in the sample (because the OLS

\footnotetext{
${ }^{16}$ These values are the marginal probability that the $R^{2}$ for a single equation exceeds 0.02 .
} 
value is "too high"). When this occurs, the predictor variable tends to have an unusually low autocorrelation; thus the OLS estimate for $\theta_{1}$ is too low and the posterior mean will be above the OLS value. Therefore, placing a prior that weights the posterior mean of $\beta$ toward zero raises the posterior mean of $\theta_{1} \cdot{ }^{17}$

Table 1 also reports posterior means and standard deviations for the means of the predictor variable and of stock returns. For example, for returns the table reports $E\left[E\left[r_{t+1} \mid B, \Sigma\right] \mid D\right]=$ $E\left[\alpha+\beta \frac{\theta_{0}}{1-\theta_{1}} \mid D\right]$ and $\left(\operatorname{Var}\left[\alpha+\beta \frac{\theta_{0}}{1-\theta_{1}} \mid D\right]\right)^{1 / 2}$. The OLS mean is set equal to $\hat{\alpha}+\hat{\beta} \frac{\hat{\theta}_{0}}{1-\hat{\theta}_{1}}$, where ${ }^{\wedge}$ denotes the OLS estimate of a parameter. The unconditional means are of interest because they help determine the average level of the portfolio allocation. The Bayesian approach implies about the same unconditional mean for the dividend-price ratio, regardless of the prior. This is close to -3.50 , the mean in the data. However, the mean implied by OLS is -3.72 . The reason that the Bayesian approach is able to identify this mean is the presence of the unconditional distribution term in the likelihood.

These differences in the mean of $x$ translate into differences in the unconditional means for returns. Table 1 shows that for the stock index, the posterior mean equals $1.17 \%$, while the OLS value is $1.09 \%$ per quarter. The sample mean for stocks in this time period was $1.67 \%$. The difference between the OLS and the sample mean arises mechanically from the difference between $\frac{\hat{\theta}_{0}}{1-\hat{\theta}_{1}}$ (equal to -3.72 ), and the sample mean of the dividend-price ratio (equal to -3.50). The difference between the sample and the Bayesian posterior mean occurs for a more subtle reason. Because the dividend-price ratio in 1952 is above its conditional maximum likelihood estimate (-3.72), it follows that shocks to the dividend-price ratio were negative on average during the time period. Because of the negative correlation between the stock return and the dividend-price ratio, shocks to stock returns must be positive on average. The exact likelihood function therefore implies a posterior mean that is below the sample mean. Similar reasoning holds for bond returns, though here, the effect is much smaller because of the low correlation between the dividend-price ratio and bond returns. This effect is not connected with the ability of the dividend-price ratio to predict returns, as it operates equally for all values of the prior.

Table 2 repeats this analysis when the yield spread is the predictor variable. The yield spread predicts both bond and stock returns with a positive sign. As the prior becomes more

\footnotetext{
${ }^{17}$ The value for $\beta$ under the diffuse prior is also substantially below the OLS value, and the value for $\theta_{1}$ is higher. The reason is that, relative to the flat prior, the Jeffreys prior favors higher values of $\theta_{1}$.
} 
diffuse, the posterior mean of the $\beta$ coefficients go from from 0 to the OLS estimate. As in the case of the dividend-price ratio, both the posterior mean of long-run expected returns and the long-run mean of $x_{t}$ are nearly the same across the range of prior distributions.

We now examine the consequences of these posterior means for the predictive distribution of returns and for portfolio choice. Figure 4 plots expected excess returns (top two plots) and optimal portfolio holdings (bottom two plots) as functions of the log dividend-price ratio. Graphs are centered at the sample mean. Diamonds denote plus and minus one and two sample standard deviations of the dividend-price ratio. We report results for the four prior beliefs discussed above; to save notation we let $P_{.02}=P\left(R^{2}>.02\right)$.

The linear form of (1) implies that expected returns are linear in the predictor variables, conditional on the past data. The slope of the relation between the conditional return and $x_{t}$ equals the posterior mean of $\beta$. Figure 4 shows large deviations in the expected return on the stock on the basis of the dividend-price ratio. As the dividend-price ratio varies from -2 standard deviations to +2 standard deviations, the expected return varies from $0 \%$ per quarter to $2 \%$ per quarter. On the other hand, the dividend-price ratio has virtually no predictive power for returns on the long-term bond.

The bottom panel of Figure 4 shows that the weight on the stock index also increases in the dividend-price ratio. Bond weights decrease in the dividend-price ratio because bond and stock returns are positively correlated, so an increase in the mean of the stock return, without a corresponding increase in the bond return, results in an optimal portfolio that puts less weight on the bond.

For the diffuse prior, weights on the stock index vary substantially, from $-30 \%$ when the dividend-price ratio is two standard deviations below its mean to $100 \%$ when the dividendprice ratio is two standard deviations above its mean. As the prior becomes more informative, expected returns and weights both vary less. However, this change happens quite slowly. Conditional expected returns under a prior that assigns only a .075 chance of an $R^{2}$ greater than $2 \%$ are nearly identical to conditional expected returns with a diffuse prior. There is sufficient evidence to convince even this skeptical investor to vary her portfolio to nearly the same degree as an investor with no skepticism at all. For a more skeptical prior with $P_{.02}=.0005$, differences emerge: the slope of the relation between expected returns and the dividend-price ratio is about half of what it was with a diffuse prior.

Figure 5 displays analogous plots for the yield spread. Both the conditional expected 
bond return and the stock return increase substantially in the yield spread. For bonds, these expected returns vary between $-2 \%$ and $2 \%$ per quarter as the yield spread varies between -2 and +2 standard deviations. For stocks, expected returns vary between $0 \%$ and $3 \%$, similar to the variation with respect to the dividend-price ratio. These large variations in expected returns lead to similarly large variation in weights for the diffuse prior: for bonds, the weights vary between $-200 \%$ and $200 \%$ as the yield spread varies between -2 and +2 standard deviations from the mean. For the stock, the weights vary between 0 and $75 \%$. The variation in the weights on the stock appears less than the variation in expected returns on the stock; this is due to the positive correlation in return innovations between stocks and bonds.

Figure 5 also shows that the more informative the prior, the less variable the weights. However, when the predictor variable is the yield spread, inference based on a skeptical prior with $P_{.02}=.075 \%$ differs noticeably from inference based on a diffuse prior. Nonetheless, even the investors with skeptical priors choose portfolios that vary with the yield spread.

This section has shown that an investor who is skeptical about predictability, when confronted with historical data, does indeed choose to time the market. The next two sections show the consequences of this for the time series of portfolio weights and for out-ofsample performance.

\subsection{Posterior means and asset allocation over the post-war period}

We next describe the implications of various prior beliefs for optimal weights over the postwar period. Starting in 1972, we compute the posterior distribution conditional on having observed data up to and including that year. We start in 1972 because this allows for twenty years of data for the first observation; this seems reasonable given the persistence in the data and the fact that there are 12 parameters to be estimated. Starting the analysis in 1982 with thirty years of data leads to very similar results. ${ }^{18}$ The posterior is computed by simulating 200,000 draws and dropping the first 50,000. Each quarter, the investor updates the portfolio weights using that quarter's observation of the predictor variable. The assets

\footnotetext{
${ }^{18}$ We update the posterior distribution yearly rather than quarterly to save on computation time, which is a particular concern when we assess the significance of our results using a Monte Carlo procedure. The results from updating quarterly are very close to those from updating yearly and are available from the authors upon request.
} 
are the stock, the long-term bond, and a riskless asset.

Figure 6 displays the de-meaned dividend-price ratio and the weights in the long-term bond and the stock for the most diffuse prior. For most of the sample, the weights in the stock are highly positively correlated with the dividend-price ratio. Less correlation is apparent for bond returns. From the mid-90's, on, this correlation is reduced for both assets: despite the continued decline in the dividend-price ratio, the allocation to stocks levels off and the allocation to bonds rises. As Figure 6 shows, under diffuse priors, portfolio weights are highly variable and often extreme.

Figure 7 offers another perspective on the relation between the predictor variable and the allocation. The top panel of the figure shows the posterior mean of $\beta$ for the stock index. The posterior means are shown for priors ranging from dogmatic to diffuse $\left(P_{.02}\right.$ ranging from 0 to 1) and for the point estimates of $\beta$ from OLS. The top panel shows that the OLS beta lies above the posterior mean for the entire sample. The OLS estimates, the posterior mean when $P_{.02}=.999$, and the posterior mean when $P_{.02}=0.075$ decline around 1995 , and then rise again around $2000 .^{19}$

The posterior mean for $P_{.02}=0.075$ lies above the posterior mean for the diffuse prior after 2000. This may seem surprising, as the role of the prior is to shrink the $\beta$ s toward zero. However, the prior shrinks the total amount of predictability as measured by the $R^{2}$. This can be accomplished not only by shrinking $\beta$, but also by shrinking the persistence relative to the diffuse prior. In contrast to the posterior means for the less informative priors, the posterior mean for $P_{.02}=.0005$ remains steady throughout the sample and actually increases after 2000 .

The bottom panel of Figure 7 shows holdings in the stock for a range of beliefs about predictability. Also displayed are holdings resulting from OLS estimation. Volatility in holdings for the stock decline substantially as the prior becomes more dogmatic. For the fully dogmatic prior, the weight on the stock index displays some initial volatility, and then stays at about $40 \%$ after about 1976. The prior that is close to dogmatic, $P_{.02}=.0005$, implies some market timing for the stock portfolio based on the dividend-price ratio. In the early part of the sample the weight in the stock index implied by this prior is about $50 \%$, declining to zero at the end of the sample. Of course, $P_{.02}=.075$ and the diffuse prior imply

\footnotetext{
${ }^{19}$ This plot is suggestive of parameter instability in the postwar sample; indeed evidence of such instability is found by Lettau and VanNieuwerburgh (2006), Paye and Timmermann (2005), and Viceira (1996).
} 
greater amounts of market timing. These priors imply time-varying weights that fluctuate both at a very slow frequency, and at a higher frequency. However, the weights that display the most volatility are those arising from ordinary least squares regression.

Figure 8 shows corresponding results for the bond. The top panel shows that for most of the sample, the dividend yield predicts bond returns with a positive sign, just as it does with stock returns. Starting in the late-90s, this predictability begins to decline. Volatility in bond holdings also decline substantially as the prior becomes more dogmatic. Corresponding figures for the yield spread (Figures 9-11) share these features.

Figure 7 demonstrates that using predictive variables in portfolio allocations need not lead to extreme weights. Combining the sample evidence with priors that are skeptical about return predictability leads to a moderate amount of market timing. We now turn to the out-of-sample performance of these strategies.

\subsection{Out-of-sample performance}

The previous sections show how skeptical priors can inform portfolio selection. The results indicate that investors who are highly skeptical about return predictability nonetheless choose time-varying weights, but that these weights are less variable and extreme than the weights for investors with diffuse priors. In this section, we assess out-of-sample performance of these priors. ${ }^{20}$ The goal is not to find out which prior is "best"; indeed this analysis should not be interpreted as suggesting that one prior is superior to another. Rather, the purpose of this section is to relate our findings to those in papers that critique the evidence for predictability based on out-of-sample performance (Bossaerts and Hillion (1999), Goyal and Welch (2004)).

To assess out-of-sample performance in a way that controls for risk, we adopt a cer-

${ }^{20}$ Avramov (2004) and Handa and Tiwari (2006) also highlight how informative non-dogmatic priors can improve ex post performance of portfolios. Unlike the present study, these studies use informative priors for the cross-section (in the style of Pastor and Stambaugh (1999)), and show that shrinking a broad cross-section of returns toward what would be implied by asset pricing models can lead to better ex post performance in settings that include return predictability. As discussed in Section 1.2, Avramov also examines the effects of introducing a "prior" sample with no predictability and no mispricing relative to the 3-factor model. When there is such a sample, performance is often better than when there is no such prior sample. However, it is not clear whether these results are due to the prior over predictability or mispricing. 
tainty equivalent approach. The certainty equivalent return (CER) answers the question: "what riskless rate would the investor be willing to accept in exchange for not following this strategy?" That is

$$
\mathrm{CER}=E\left[r_{p}\right]-A \frac{1}{2} \operatorname{Var}\left[r_{p}\right]
$$

where $A$ is the appropriate risk-aversion parameter (see, e.g., Brennan and Xia (2001)). In this analysis, the mean and variance in (20) are computed using the sample mean and variance that result from following strategies associated with a given prior belief. That is, for each quarter, we apply the weights described in the previous section to the actual returns realized over the next quarter. This gives us a time series of 120 quarterly returns to use in computing the means and variances. In reporting the certainty equivalent returns, we multiply by 400 to express the return as an annual percentage. Results are reported for values of $A$ equal to 2 and 5 . As an additional metric, we also report out-of-sample Sharpe ratios. These are equal to the sample mean of excess returns, divided by the sample standard deviation. Excess returns are quarterly and constructed as described above. In reporting Sharpe ratios, we multiply by 2 to annualize.

Table 3 reports CERs and Sharpe ratios when the dividend-price ratio is the predictor variable. For both metrics, the weights implied by ordinary least squares deliver worse performance than the weights implied by the dogmatic prior, which implies no market timing. A similar result is found by Goyal and Welch (2004), who argue against the use of predictability in portfolio allocation. We find, however, that market timing can increase out-of-sample performance, if the investor treats the evidence with some skepticism. The intermediate prior with $P_{.02}=.0005$ has the best out-of-sample performance regardless of the level of risk aversion and whether the CER or Sharpe ratio metric is used. While ignoring the predictability evidence results in better performance than applying a diffuse prior, simply looking at these two extreme positions hides the better performance that can be achieved by taking the intermediate view.

Table 4 reports analogous results for the yield spread as the predictor variable. For the certainty equivalent return metric, OLS again performs the worst, while the diffuse and dogmatic priors perform somewhat better. However, skeptical priors perform better still. ${ }^{21}$ The differences can be large. When the risk aversion parameter equals 2 , for example, a

\footnotetext{
${ }^{21}$ For the Sharpe ratio metric, OLS and the diffuse priors also outperform the most dogmatic prior.
} 
diffuse prior results in a CER of $5.47 \%$ per annum, while a dogmatic prior results in a CER of $5.61 \%$. However, for the intermediate prior of $P_{.02}=.0005$, the CER is $7.58 \%$ per annum; for the prior with $P_{.02}=.075$, the CER is $9.19 \%$ per annum. These results show that priors indicating skepticism about the degree of predictability lead to superior out-of-sample performance over the postwar period, as well as to less extreme portfolio allocations, and to more stable parameter estimates.

We next evaluate the classical statistical significance and robustness of our out-of-sample results. The analysis in the previous sections indicates that a Bayesian investor, even one who is skeptical about return predictability, would time the market. There is sufficient evidence to convince this investor that market timing is worthwhile. Here however we ask a different question. Suppose that returns are in fact unpredictable. In repeated samples, what is the probability that our strategies would have led to (presumably spurious) superior performance?

To answer this question, we use a Monte Carlo procedure to correctly capture the dependencies inherent in our out-of-sample return observations (even though the shocks to returns and the predictor variable may be independent across time, portfolio returns depend on the investor's posterior, and therefore in principle on all previous observations). We simulate 100 samples of data designed so that means, variances, and covariances of returns and of the state variable, and the autocorrelation of the state variable are the same as in the data. However, in simulated data, returns are unpredictable. We then calculate out-of-sample performance, exactly repeating the procedure we used to calculate performance in actual data. $^{22}$

Table 5 shows the results of this exercise for the dividend-price ratio. The first row of Panel A reports the difference between the CER of each skeptical prior (and the regression estimate) and that of the dogmatic prior seen in the data for a risk aversion of 2 . These differences are a measure of the ex-post gains from market timing. The next two rows report

\footnotetext{
${ }^{22}$ We consider only 100 draws because of the heavy computational requirements. While sampling from the posterior distribution for a given dataset takes under an hour, obtaining out-of-sample statistics requires that this procedure be repeated 32 times (once for every sample year since 1972). The whole procedure then needs to be repeated 100 times for the Monte Carlo. The entire set of Monte Carlo results (for two predictor variables and four priors) requires about a month of computation time on a 10-node Beowulf cluster. This prevents us from embarking on more detailed statistical analyses, e.g. correcting for the impact of searching over the two skeptical priors.
} 
the 5th and the 95th percentile of the Monte-Carlo-generated statistic. The results show that the outperformance of the prior $P_{.02}=.0005$ could have occurred by chance with at most a $10 \%$ probability using a two-tailed test (because computation costs force us to limit the Monte Carlo to 100 draws, critical values corresponding to higher levels of significance would be unreliable). In the data, outperformance is 69 basis points per year while the 95th percentile is 61 basis points. Panel B reports the results of a similar exercise for the Sharpe ratio. The difference in the data between the skeptical and the dogmatic prior is 0.025 ; the 95th percentile is only 0.007 .

Table 6 reports analogous results for the yield spread. Both skeptical priors $\left(P_{.02}=.0005\right.$ and $\left.P_{.02}=.075\right)$ outperform the dogmatic prior. This outperformance is far above the 95th percentile for the Monte Carlo draws for both the CER and the Sharpe ratio measure. For example, when risk aversion is $2, P_{.02}=.075$ implies a performance differential of $3.58 \%$. The 95th percentile is $1.57 \%$. When risk aversion is 5 , this prior implies a performance differential of $1.46 \%$; the 95 th percentile is $0.63 \%$. Taken together, our Monte Carlo results suggest that it is quite unlikely that the superior performance of the skeptical priors could have occurred by chance.

Finally, we examine the performance of our strategies over subperiods of the data. The results above use data from 1952 to 2004. Here, we repeat the analysis with data ending in 1984 and 1994. The results for the dividend-price ratio are shown in Table 7. For each subsample, OLS continues to give the worst performance as measured by certainty equivalent returns. However, skeptical priors deliver superior performance relative to the dogmatic prior. Similar results hold when the yield spread is the predictor variable, as shown in Table 8. In both subsamples, and across all measures of performance, the skeptical priors outperform the dogmatic prior.

To conclude, we find that market timing strategies implied by OLS underperform strategies implied by a no-predictability view, confirming results in other recent papers. However, we also find that taking an intermediate view, that some predictability might exist, leads to better performance relative to either extreme. 


\section{Extensions to multiple predictor variables}

This section extends the results in Section 1 to the case of multiple predictor variables. We continue to assume (1) and (2), except that $x_{t}$ is now allowed to be $K \times 1, \beta$ is $N \times K, \theta_{0}$ is $K \times 1$, and $\theta_{1}$ is $K \times K$. Define

$$
B=\left[\begin{array}{ll}
\alpha^{\top} & \theta_{0}^{\top} \\
\beta^{\top} & \theta_{1}^{\top}
\end{array}\right]
$$

The multivariate analogues of (5) and (6) are

$$
\mu_{x}=E\left[x_{t} \mid B, \Sigma\right]=\left(I_{K}-\theta_{1}\right)^{-1} \theta_{0}
$$

and

$$
\operatorname{vec}\left(\Sigma_{x}\right)=\left(I_{K^{2}}-\left(\theta_{1} \otimes \theta_{1}\right)\right)^{-1} \operatorname{vec}\left(\Sigma_{v}\right)
$$

where $\Sigma_{x}=E\left[\left(x_{t}-x_{0}\right)\left(x_{t}-x_{0}\right)^{\top}\right]$ (see Hamilton $(1994$, p. 265)).

As in Section 1, we specify a prior over normalized $\beta$. Let $C_{x}$ be a lower triangular matrix such that $C_{x} C_{x}^{\top}=\Sigma_{x}$, and

$$
\eta=C_{u}^{-1} \beta C_{x}
$$

(recall that $C_{u}$ is such that $C_{u} C_{u}^{\top}=\Sigma_{u}$ ). Assume prior beliefs on $\eta$ are given by

$$
\operatorname{vec}(\eta) \sim N\left(0, \sigma_{\eta}^{2} I_{N K}\right)
$$

Then (23) implies

$$
\operatorname{vec}(\eta)=\left(C_{x}^{\top} \otimes C_{u}^{-1}\right) \operatorname{vec}(\beta)
$$

and therefore,

$$
\operatorname{vec}(\beta) \mid \alpha, \theta_{0}, \theta_{1}, \Sigma \sim N\left(0, \sigma_{\eta}^{2}\left(\Sigma_{x}^{-1} \otimes \Sigma_{u}\right)\right) .
$$

Appendix $\mathrm{C}$ shows that the Jeffreys prior on $\left(\alpha, \theta_{0}, \theta_{1}, \Sigma\right)$ takes the form

$$
p\left(\alpha, \theta_{0}, \theta_{1} \Sigma\right) \propto|\Sigma|^{-\frac{N+2 K+2}{2}}\left|\Sigma_{u}\right|^{K / 2}\left|\Sigma_{x}\right|^{K / 2} .
$$

Because

$$
\begin{aligned}
\operatorname{vec}(\beta)^{\top}\left(\Sigma_{u} \otimes \Sigma_{x}^{-1}\right)^{-1} \operatorname{vec}(\beta) & =\operatorname{vec}(\beta)^{\top}\left(\Sigma_{u}^{-1} \otimes \Sigma_{x}\right) \operatorname{vec}(\beta) \\
& =\operatorname{tr}\left(\beta^{\top} \Sigma_{x} \beta \Sigma_{u}^{-1}\right),
\end{aligned}
$$


the joint prior is given by

$$
p(B, \Sigma) \propto\left|\Sigma_{x}\right|^{\frac{N+K}{2}}|\Sigma|^{-\frac{N+2 K+2}{2}} \exp \left\{-\frac{1}{2} \sigma_{\eta}^{-2} \operatorname{tr}\left(\beta^{\top} \Sigma_{x} \beta \Sigma_{u}^{-1}\right)\right\} .
$$

As $\sigma_{\eta}$ approaches zero, $p(B, \Sigma)$ approaches a dogmatic, no-predictability prior. Appendix B shows that $p(B, \Sigma)$ approaches a Jeffreys prior as $\sigma_{\eta}$ approaches infinity. Intermediate levels of $\sigma_{\eta}$ allow for skepticism about the level of predictability by placing an informative prior on the maximum $R^{2}$ implied by the equations (1) and (2). The generalization of the equation for the maximum $R^{2}$ is

$$
\begin{aligned}
\max _{w} R^{2} & =\max _{w} \frac{w^{\top} \beta \Sigma_{x} \beta^{\top}}{w^{\top} \beta \Sigma_{x} \beta^{\top} w+w^{\top} \Sigma_{u} w} \\
& =\frac{\eta^{\top} \eta}{\eta^{\top} \eta+1}
\end{aligned}
$$

as shown in Appendix A.

The computations for the likelihood and the posterior closely follow those in Section 1. Define

$$
Y=\left[\begin{array}{cc}
r_{1}^{\top} & x_{1}^{\top} \\
\vdots & \vdots \\
r_{T}^{\top} & x_{T}^{\top}
\end{array}\right], \quad X=\left[\begin{array}{cc}
1 & x_{0}^{\top} \\
\vdots & \vdots \\
1 & x_{T-1}^{\top}
\end{array}\right]
$$

The exact likelihood is given by

$$
\begin{aligned}
p(D \mid B, \Sigma)= & \left|2 \pi \Sigma_{x}\right|^{-\frac{1}{2}}|2 \pi \Sigma|^{-\frac{T}{2}} \\
& \exp \left\{-\frac{1}{2}\left(x_{0}-\mu_{x}\right)^{\top} \Sigma_{x}^{-1}\left(x_{0}-\mu_{x}\right)-\frac{1}{2} \operatorname{tr}\left[(Y-X B)^{\top}(Y-X B) \Sigma^{-1}\right]\right\} .
\end{aligned}
$$

Applying Bayes rule to (26) and (28) leads to the posterior

$$
\begin{aligned}
p(B, \Sigma \mid D) \propto\left|\Sigma_{x}\right| \frac{N+K-1}{2} & |\Sigma|^{-\frac{T+N+2 K+2}{2}} \exp \left\{-\frac{1}{2}\left(x_{0}-\mu_{x}\right)^{\top} \Sigma_{x}^{-1}\left(x_{0}-\mu_{x}\right)\right\} \\
& \exp \left\{-\frac{1}{2} \sigma_{\eta}^{-2} \operatorname{tr}\left(\beta^{\top} \Sigma_{x} \beta \Sigma_{u}^{-1}\right)-\frac{1}{2} \operatorname{tr}\left[(Y-X B)^{\top}(Y-X B) \Sigma^{-1}\right]\right\} .
\end{aligned}
$$

The procedure for sampling from this posterior is very similar to that described in Section 1.3. The Metropolis-Hastings algorithm is used to draw blocks of parameters at a time. First, $\Sigma$ is drawn from $p(\Sigma \mid B, D)$ using the inverted Wishart distribution with $T+K+1$ degrees of freedom. Second, $\alpha$ and $\beta$ are drawn from $p\left(\alpha, \beta \mid \theta_{0}, \theta_{1}, D\right)$, and finally $\theta_{0}$ and $\theta_{1}$ are drawn from $p\left(\theta_{0}, \theta_{1} \mid \alpha, \beta, \Sigma, D\right)$. The computation of the conditional posteriors for $(\alpha, \beta)$, and $\left(\theta_{0}, \theta_{1}\right)$ is exactly as in Section 1.3. 


\section{Conclusions}

How much evidence on predictability is enough to influence portfolio choice? One view is that predictability should be taken into account only if the statistical evidence for it is incontrovertible. An opposite view is that investors should time their allocations to a large extent, even if the evidence for predictability is weak according to conventional measures. The first view states that investors should be extremely skeptical when viewing data showing evidence of predictability, while the second view states that no skepticism is necessary at all.

In this paper, we modeled the portfolio choice problem of an investor who has prior beliefs on the amount of predictability in the data. These prior beliefs put "skepticism" about predictability on a sound decision-theoretic basis. The skeptical investor believes that, while predictability is possible, large values of the $R^{2}$ are unlikely.

We applied our method to post-war data on bond and stock returns, with the yield spread and the dividend-price ratio as predictor variables. We found that even investors with a high degree of prior skepticism still vary their allocations to long-term bonds and stocks based on both of these variables. Thus the amount of predictability in the data is sufficient to influence investment, even if the investor is skeptical about the strength of this predictability.

To see the implications of various prior beliefs for portfolio allocations over the postwar

period, we implemented an out-of-sample analysis. For each quarter starting in 1972, the posterior and optimal portfolio weights were determined based on previous data. Parameter estimates implied by skeptical priors were more stable than those implied by the diffuse prior and by ordinary least squares regression. Moreover, the resulting weights are less volatile and deliver superior out-of-sample performance as compared to the weights implied by an entirely model-based or data-based view.

This study provides a method for rigorously implementing an intermediate view on predictability. The resulting portfolio weights are more reasonable, and in fact perform better out of sample than either extreme view. The question remains as to why the skeptical prior outperforms. Skepticism can be motivated by a theory of rational markets (see Samuelson (1965)). Our results suggest that there may be value to this theory even if it does not hold exactly. More broadly, this study supports the idea that using models to downweight unreasonable regions of the parameter space may improve decision making. 


\section{Appendix}

\section{A Derivation of the maximum $R^{2}$}

This section derives equation (11) and (27). Because (11) is a special case of (27), we derive this latter equation. The maximum $R^{2}$ satisfies

$$
\max R^{2}=\max _{w} \frac{w^{\top} \beta \Sigma_{x} \beta^{\top} w}{w^{\top} \beta \Sigma_{x} \beta^{\top} w+w^{\top} \Sigma_{u} w}
$$

where $w$ is an $N \times 1$ nonzero vector (it is not necessary that the elements of $w$ sum to 1 ). Define

$$
\bar{w}=C_{u}^{\top} w
$$

where $C_{u} C_{u}^{\top}=\Sigma_{u}$. Rewrite (30) as

$$
\begin{aligned}
\max R^{2} & =\max _{\bar{w}} \frac{\bar{w}^{\top} C_{u}^{-1} \beta \Sigma_{x} \beta^{\top}\left(C_{u}^{-1}\right)^{\top} \bar{w}}{\bar{w}^{\top} C_{u}^{-1} \beta \Sigma_{x} \beta^{\top}\left(C_{u}^{-1}\right)^{\top} \bar{w}+\bar{w}^{\top} \bar{w}} \\
& =\max _{\bar{w}} \frac{\bar{w}^{\top} \eta \eta^{\top} \bar{w}}{\bar{w}^{\top} \eta \eta^{\top} \bar{w}+\bar{w}^{\top} \bar{w}} \\
& =\max _{w} \frac{w^{\top} \eta \eta^{\top} w}{w^{\top} \eta \eta^{\top} w+w^{\top} w}
\end{aligned}
$$

where the last line follows from a change of notation for the maximizing variable. Finally, we note that

$$
\operatorname{argmax}_{w} \frac{w^{\top} \eta \eta^{\top} w}{w \eta \eta^{\top} w+w^{\top} w}=\operatorname{argmax}_{w} \frac{\frac{w^{\top} \eta \eta^{\top} w}{w^{\top} w}}{\frac{w \eta \eta^{\top} w}{w^{\top} w}+1}=\operatorname{argmax}_{w} \frac{w^{\top} \eta \eta^{\top} w}{w^{\top} w} .
$$

because the argument in the former two expressions is an increasing function of the last expression. By the Cauchy-Schwartz inequality,

$$
\frac{w^{\top} \eta \eta^{\top} w}{w^{\top} w} \leq \eta^{\top} \eta
$$

for all nonzero vectors $w$, and therefore

$$
\eta=\operatorname{argmax}_{w} \frac{w^{\top} \eta \eta^{\top} w}{w \eta \eta^{\top} w+w^{\top} w} .
$$

This proves (27). 


\section{B Jeffreys prior on $B, \Sigma$}

Our derivation for the limiting Jeffreys prior on $B, \Sigma$ follows Stambaugh (1999). Zellner (1996, pp. 216-220) derives a limiting Jeffreys prior by applying (12) to the likelihood (7) and retaining terms of the highest order in $T$. Stambaugh shows that Zellner's approach is equivalent to applying (12) to the conditional likelihood (4), and taking the expectation in (12) assuming that $x_{0}$ is multivariate normal with mean (21) and variance (22) (or mean (5) and variance (6) in the $K=1$ case). We adopt this approach.

We derive the prior density for $p\left(B, \Sigma^{-1}\right)$ and then transform this into the density for $p(B, \Sigma)$ using the Jacobian. Let $b=\operatorname{vec}(B)$, and

$$
\zeta=\left(\sigma^{11}, \sigma^{12}, \ldots, \sigma^{1, N+K}, \sigma^{22}, \sigma^{23}, \ldots, \sigma^{2, N+K}, \ldots, \sigma^{N+K, N+K}\right),
$$

where $\sigma^{i j}$ denotes element $(i, j)$ of $\Sigma^{-1}$. Let $l(B, \Sigma ; D)=\log p\left(D \mid B, \Sigma, x_{0}\right)$ denote the natural $\log$ of the conditional likelihood. The definition of the Jeffreys prior requires

$$
p\left(B, \Sigma^{-1}\right) \propto\left|-E\left(\left[\begin{array}{cc}
\frac{\partial^{2} l}{\partial b \partial b^{\top}} & \frac{\partial^{2} l}{\partial b \partial \zeta^{\top}} \\
\frac{\partial^{2} l}{\partial \zeta \partial b^{\top}} & \frac{\partial^{2} l}{\partial \zeta \partial \zeta^{\top}}
\end{array}\right]\right)\right|^{1 / 2}
$$

Computing the expectation on the right hand side of (31) yields

$$
p\left(B, \Sigma^{-1}\right) \propto\left|\begin{array}{cc}
\Sigma^{-1} \otimes \Psi & 0 \\
0 & \frac{\partial^{2} \log |\Sigma|}{\partial \zeta \partial \zeta^{\top}}
\end{array}\right|^{1 / 2},
$$

where

$$
\Psi=\left[\begin{array}{cc}
1 & \mu_{x}^{\top} \\
\mu_{x} & \mu_{x} \mu_{x}^{\top}+\Sigma_{x}
\end{array}\right]
$$

Note that $\Psi$ is $(K+1) \times(K+1)$. From the formula for the determinant of a partitioned matrix (Green (1997, p. 33)), it follows that $|\Psi|=\Sigma_{x}$.

Box and Tiao (1973, pp. 474-475) show that

$$
\left|\frac{\partial^{2} \log |\Sigma|}{\partial \zeta \partial \zeta^{\top}}\right|^{1 / 2}=|\Sigma|^{\frac{N+K+1}{2}}
$$

(recall that $\Sigma$ is $(N+K) \times(N+K))$. It then follows from (32) that

$$
\begin{aligned}
p\left(B, \Sigma^{-1}\right) & \propto\left|\Sigma^{-1} \otimes \Psi\right|^{1 / 2}|\Sigma|^{\frac{N+K+1}{2}} \\
& =\left(|\Sigma|^{-(K+1)}|\Psi|^{N+K}\right)^{1 / 2}|\Sigma|^{\frac{N+K+1}{2}} \\
& =|\Psi|^{\frac{N+K}{2}}|\Sigma|^{N / 2} \\
& =\left|\Sigma_{x}\right|^{\frac{N+K}{2}}|\Sigma|^{N / 2} .
\end{aligned}
$$


From results in Zellner (1996, p. 226), it follows that Jacobian of the transformation from $\Sigma^{-1}$ to $\Sigma$ is $|\Sigma|^{-(N+K+1)}$. Therefore

$$
p(B, \Sigma) \propto\left|\Sigma_{x}\right|^{\frac{N+1}{2}}|\Sigma|^{-\frac{N+2 K+2}{2}} .
$$

\section{Jeffreys prior on $\alpha, \theta_{0}, \theta_{1}, \Sigma$}

We calculate the prior for $\left(\alpha, \theta_{0}, \theta_{1}, \Sigma^{-1}\right)$, and use the determinant of the Jacobian to transform this into a prior for $\left(\alpha, \theta_{0}, \theta_{1}, \Sigma\right)$. Define blocks of $\Sigma^{-1}$ as

$$
\Sigma^{-1}=\left[\begin{array}{cc}
\left(\Sigma^{-1}\right)_{11} & \left(\Sigma^{-1}\right)_{12} \\
\left(\Sigma^{-1}\right)_{21} & \left(\Sigma^{-1}\right)_{22}
\end{array}\right]
$$

Here, $\left(\Sigma^{-1}\right)_{11}$ is $N \times N,\left(\Sigma^{-1}\right)_{12}$ is $N \times K,\left(\Sigma^{-1}\right)_{21}=\left(\Sigma^{-1}\right)_{12}^{\top}$, and $\left(\Sigma^{-1}\right)_{22}$ is $K \times K$.

The starting point for the calculation is the information matrix (12) for $B, \Sigma^{-1}$ given in Appendix B. The information matrix for $\alpha, \theta_{0}, \theta_{1}, \Sigma^{-1}$ can be obtained by removing the rows and columns corresponding to derivatives with respect to $\beta_{i j}$ from (32). Without loss of generality, the rows and columns of (32) can be re-ordered so that

$$
p\left(B, \Sigma^{-1}\right) \propto\left|\begin{array}{cc}
\Psi \otimes \Sigma^{-1} & 0 \\
0 & \frac{\partial^{2} \log |\Sigma|}{\partial \zeta \partial \zeta^{\top}}
\end{array}\right|^{1 / 2}
$$

This corresponds to taking second derivatives of $l$ with respect to $\operatorname{vec}\left(B^{\top}\right)$ rather than $\operatorname{vec}(B)$. Because $\operatorname{vec}\left(B^{\top}\right)=\left(\alpha^{\top}, \theta_{0}^{\top}, \operatorname{vec}(\beta)^{\top}, \operatorname{vec}\left(\theta_{1}\right)^{\top}\right)^{\top}$, removing the rows and columns corresponding to $\operatorname{vec}(\beta)$ leads to

$$
p\left(\alpha, \theta_{0}, \theta_{1}, \Sigma^{-1}\right) \propto\left|\begin{array}{cc}
\Phi & 0 \\
0 & \frac{\partial^{2} \log |\Sigma|}{\partial \zeta \partial \zeta^{\top}}
\end{array}\right|^{1 / 2}
$$

where

$$
\Phi=\left[\begin{array}{cc}
\Sigma^{-1} & \mu_{x}^{\top} \otimes\left[\begin{array}{c}
\left(\Sigma^{-1}\right)_{12} \\
\left(\Sigma^{-1}\right)_{22}
\end{array}\right] \\
\mu_{x} \otimes\left[\left(\Sigma^{-1}\right)_{21},\left(\Sigma^{-1}\right)_{22}\right] & \left(\Sigma_{x}+\mu_{x} \mu_{x}^{\top}\right) \otimes\left(\Sigma^{-1}\right)_{22}
\end{array}\right] .
$$

From the formula for the determinant of a partitioned matrix, it follows that $|\Phi|=\left|\Sigma^{-1}\right|\left|\left(\Sigma_{x}+\mu_{x} \mu_{x}^{\top}\right) \otimes\left(\Sigma^{-1}\right)_{22}-\left(\mu_{x} \otimes\left[\left(\Sigma^{-1}\right)_{21},\left(\Sigma^{-1}\right)_{22}\right]\right) \Sigma\left(\mu_{x}^{\top} \otimes\left[\begin{array}{c}\left(\Sigma^{-1}\right)_{12} \\ \left(\Sigma^{-1}\right)_{22}\end{array}\right]\right)\right|$. 
Because

$$
\Sigma\left[\begin{array}{c}
\left(\Sigma^{-1}\right)_{12} \\
\left(\Sigma^{-1}\right)_{22}
\end{array}\right]=\left[\begin{array}{c}
0_{N \times K} \\
I_{K}
\end{array}\right]
$$

it follows that

$$
\begin{aligned}
|\Phi| & =\left|\Sigma^{-1}\right|\left|\left(\Sigma_{x}+\mu_{x} \mu_{x}^{\top}\right) \otimes\left(\Sigma^{-1}\right)_{22}-\mu_{x} \mu_{x}^{\top} \otimes\left(\Sigma^{-1}\right)_{22}\right| \\
& =\left|\Sigma^{-1}\right|\left|\Sigma_{x} \otimes\left(\Sigma^{-1}\right)_{22}\right| \\
& =\left.\left|\Sigma^{-1}\right| \Sigma_{x}\right|^{K}\left|\left(\Sigma^{-1}\right)_{22}\right|^{K} .
\end{aligned}
$$

Applying the formula for the determinant of a partitioned matrix to $\Sigma$ produces

$$
|\Sigma|=\left|\Sigma_{u}\right|\left|\Sigma_{v}-\Sigma_{v u} \Sigma_{u}^{-1} \Sigma_{u v}\right|
$$

By the formula for the inverse of a partitioned matrix (Green (1997, p. 33)),

$$
\left|\left(\Sigma^{-1}\right)_{22}\right|=\left|\Sigma_{v}-\Sigma_{v u} \Sigma_{u}^{-1} \Sigma_{u v}\right|^{-1}
$$

Therefore,

$$
\begin{aligned}
|\Phi| & =|\Sigma|^{-(K+1)}|\Sigma|^{K}\left|\left(\Sigma^{-1}\right)_{22}\right|^{K}\left|\Sigma_{x}\right|^{K} \\
& =|\Sigma|^{-(K+1)}\left|\Sigma_{u}\right|^{K}\left|\Sigma_{x}\right|^{K} .
\end{aligned}
$$

Finally, from (33) and (35),

$$
\begin{aligned}
p\left(\alpha, \theta_{0}, \theta_{1}, \Sigma^{-1}\right) & \propto|\Phi|^{1 / 2}|\Sigma|^{\frac{N+K+1}{2}} \\
& =|\Sigma|^{N / 2}\left|\Sigma_{u}\right|^{K / 2}\left|\Sigma_{x}\right|^{K / 2}
\end{aligned}
$$

which, by the Jacobian of the transformation from $\Sigma^{-1}$ to $\Sigma$ (see Appendix B) completes the proof of $(25)$.

\section{Sampling from the posterior}

The conditional density $p(\Sigma \mid B, D)$ has an expression identical to (16). The proposal density is the inverted Wishart with $T+2$ degrees of freedom (Zellner (1996, p. 395)):

$$
f(\Sigma \mid B, D) \propto|\Sigma|^{-\frac{T+N+2}{2}} \exp \left\{-\frac{1}{2} \operatorname{tr}\left[(Y-X B)^{\top}(Y-X B) \Sigma^{-1}\right]\right\} .
$$


Because the target density takes the form

$$
p(\Sigma \mid B, D) \propto \psi(\Sigma) \times \text { proposal }
$$

we can use the results in Chib and Greenberg (1995, Section 5) to sample from the posterior.

The density for $p(B \mid \Sigma, D)$ is sampled from in two steps: first we sample from $p\left(b_{1} \mid b_{2}, \Sigma, D\right)$, and next we sample from $p\left(b_{2} \mid b_{1}, \Sigma, D\right)$. For the first of these steps, we can sample directly from the true density, without using the accept-reject algorithm. Note that $\operatorname{tr}\left[(Y-X B)^{\top}(Y-X B) \Sigma^{-1}\right]=(b-\hat{b})^{\top}\left(\Sigma^{-1} \otimes X^{\top} X\right)(b-\hat{b})+$ terms independent of $B$ where $\hat{b}=\operatorname{vec}(\hat{B})$, and $\hat{B}=\left(X^{\top} X\right)^{-1} X^{\top} Y$. Let $V=\left(\Sigma^{-1} \otimes X^{\top} X\right)^{-1}$, and partition $V$ so that

$$
V=\left[\begin{array}{ll}
V_{11} & V_{12} \\
V_{21} & V_{22}
\end{array}\right],
$$

where $V_{11}$ is $2 N \times 2 N, V_{12}$ is $2 N \times 2$, and $V_{22}$ is $2 \times 2$. Then

$$
\begin{array}{r}
(b-\hat{b})^{\top}\left(\Sigma^{-1} \otimes X^{\top} X\right)(b-\hat{b})= \\
\begin{array}{r}
\left(b_{1}-\hat{b}_{1}-V_{21}^{\top} V_{22}^{-1}\left(b_{2}-\hat{b}_{2}\right)\right)^{\top}\left(V_{11}-V_{21}^{\top} V_{22}^{-1} V_{21}\right)^{-1}\left(b_{1}-\hat{b}_{1}-V_{21}^{\top} V_{22}^{-1}\left(b_{2}-\hat{b}_{2}\right)\right) \\
\text { + terms independent of } b_{1} .
\end{array}
\end{array}
$$

(see Green (1997, Chapter 3.1)). Then under the prior (14),

$$
\begin{aligned}
& p\left(b_{1} \mid b_{2}, \Sigma, D\right) \propto \exp \left\{-\frac{1}{2} \beta^{\top}\left(\sigma_{\eta}^{2} \sigma_{x}^{-2} \Sigma_{u}\right)^{-1} \beta\right\} \\
& \exp \left\{-\frac{1}{2}\left(b_{1}-\hat{b}_{1}-V_{21}^{\top} V_{22}^{-1}\left(b_{2}-\hat{b}_{2}\right)\right)^{\top}\left(V_{11}-V_{21}^{\top} V_{22}^{-1} V_{21}\right)^{-1}\left(b_{1}-\hat{b}_{1}-V_{21}^{\top} V_{22}^{-1}\left(b_{2}-\hat{b}_{2}\right)\right)\right\} .
\end{aligned}
$$

We now complete the square to derive the distribution for $b_{1}$. Let

$$
\Omega=\Sigma_{u}^{-1} \otimes\left[\begin{array}{cc}
0 & 0 \\
0 & \left(\sigma_{\eta}^{2} \sigma_{x}^{-2}\right)^{-1}
\end{array}\right] .
$$

The posterior distribution for $b_{1}$ conditional on $b_{2}$ and $\Sigma$ is normal with variance

$$
V_{11}^{*}=\left(\Omega+\left(V_{11}-V_{12} V_{22}^{-1} V_{12}\right)^{-1}\right)^{-1}
$$

and mean

$$
b_{1}^{*}=V_{11}^{*}\left(V_{11}-V_{12} V_{22}^{-1} V_{12}\right)^{-1}\left(\hat{b}_{1}+V_{21}^{\top} V_{22}^{-1}\left(b_{2}-\hat{b}_{2}\right)\right)
$$


Similar reasoning can be used to draw from the posterior for $b_{2}=\left[\theta_{0}, \theta_{1}\right]$ conditional on the other parameters, Applying the results of Green (1997, Chapter 3.1),

$$
\begin{aligned}
& (b-\hat{b})^{\top}\left(\Sigma^{-1} \otimes X^{\top} X\right)(b-\hat{b})= \\
& \begin{array}{r}
\left(b_{2}-\hat{b}_{2}-V_{12}^{\top} V_{11}^{-1}\left(b_{1}-\hat{b}_{1}\right)\right)^{\top}\left(V_{22}-V_{12}^{\top} V_{11}^{-1} V_{12}\right)^{-1}\left(b_{2}-\hat{b}_{2}-V_{12}^{\top} V_{11}^{-1}\left(b_{1}-\hat{b}_{1}\right)\right) \\
\text { + terms independent of } b_{2} .
\end{array}
\end{aligned}
$$

The density is sampled from using an accept-reject algorithm. The proposal density is normal with mean $\hat{b}_{2}+V_{12}^{\top} V_{11}^{-1}\left(b_{1}-\hat{b}_{1}\right)$ and variance $V_{22}-V_{12}^{\top} V_{11}^{-1} V_{12}$. The target density is

$$
p\left(b_{2} \mid b_{1}, \Sigma, D\right) \propto \sigma_{x}^{N} \exp \left\{-\frac{1}{2} \beta^{\top}\left(\sigma_{\eta}^{2} \sigma_{x}^{-2} \Sigma_{u}\right)^{-1} \beta-\frac{1}{2} \sigma_{x}^{-2}\left(x_{0}-\mu_{x}\right)^{2}\right\} \times \text { proposal. }
$$

for $\theta_{1} \in(0,1)$ and zero otherwise. ${ }^{23}$

\footnotetext{
${ }^{23}$ For the case of multiple predictor variables, the procedure for simulating from the posterior is very similar. In this case, $V_{11}$ is $N K \times N K$ and

$$
\Omega=\Sigma_{u}^{-1} \otimes\left[\begin{array}{cc}
0 & 0 \\
0 & \sigma_{\eta}^{-2} \Sigma_{x}
\end{array}\right] .
$$
}

The proposal density for $\Sigma$ is an inverted Wishart with $T+K+1$ degrees of freedom. 


\section{References}

Abel, Andrew, 1990, Asset prices under habit formation and catching up with the Joneses, American Economic Review Papers and Proceedings 80, 38-42.

Abel, Andrew, 1999, Risk premia and term premia in general equilibrium, Journal of Monetary Economics 43, 3-33.

Amihud, Yakov, and Clifford Hurvich, 2004, Predictive regressions: a reduced-bias estimation method, forthcoming, Journal of Financial and Quantitative Analysis.

Ang, Andrew, and Geert Bekaert, 2006, Stock return predictability: Is it there?, forthcoming, Review of Financial Studies.

Avramov, Doron, 2002, Stock return predictability and model uncertainty, Journal of Financial Economics 64, 423-458.

Avramov, Doron, 2004, Stock return predictability and asset pricing models, Review of Financial Studies 17, 699-738.

Backus, David, A. Gregory, and Stanley Zin, 1989, Risk premiums in the term structure: Evidence from artificial economies, Journal of Monetary Economics 24, 371-399.

Baks, Klaas P., Andrew Metrick, and Jessica Wachter, 2001, Should investors avoid all actively managed mutual funds? A study in Bayesian performance evaluation, The Journal of Finance 56, 45-86.

Balduzzi, Pierluigi, and Anthony W. Lynch, 1999, Transaction costs and predictability: some utility cost calculations, Journal of Financial Economics 52, 47-78.

Barberis, Nicholas, 2000, Investing for the long run when returns are predictable, Journal of Finance 55, 225-264.

Bawa, Vijay S., Stephen J. Brown, and Roger W. Klein, 1979, Estimation risk and optimal portfolio choice. (North-Holland Amsterdam).

Bekaert, Geert, Robert J. Hodrick, and David A. Marshall, 1997, On biases in tests of the expectations hypothesis of the term structure of interest rates, Journal of Financial Economics 44, 309-348. 
Berger, James O., 1985, Statistical decision theory and Bayesian analysis. (Springer New York).

Best, Michael J., and Robert R. Grauer, 1991, On the sensitivity of mean-variance-efficient portfolios to changes in asset means: Some analytical and computational results, Review of Financial Studies 4, 315-342.

Black, Fischer, and Robert Litterman, 1992, Global portfolio optimization, Financial Analysts Journal pp. 28-43.

Bossaerts, Peter, and Pierre Hillion, 1999, Implementing statistical criteria to select return forecasting models: What do we learn?, Review of Financial Studies 12, 405-428.

Box, George, Gwilym M. Jenkins, and Gregory Reinsel, 1970, Time Series Analysis: Forecasting and Control. (Holden-Day Inc. S. Francisco, CA).

Box, George E.P., and George C. Tiao, 1973, Bayesian Inference in Statistical Analysis. (Addison-Wesley Pub. Co. Reading, MA).

Brandt, Michael W., Amit Goyal, Pedro Santa-Clara, and Jonathan R. Stroud, 2005, A simulation approach to dynamics portfolio choice with an application to learning about return predictability, Review of Financial Studies 18, 831-873.

Brennan, Michael J., Eduardo S. Schwartz, and Ronald Lagnado, 1997, Strategic asset allocation, Journal of Economic Dynamics and Control 21, 1377-1403.

Brennan, Michael J., and Yihong Xia, 2001, Assessing asset pricing anomalies, Review of Financial Studies 14, 905-942.

Brennan, Michael J., and Yihong Xia, 2005, Persistence, predictability, and portfolio planning, Working paper, University of California at Los Angeles.

Brown, Stephen J., 1979, Optimal portfolio choice under uncertainty: A Bayesian approach, in Vijay S. Bawa, Stephen J. Brown, and Roger W. Klein, eds.: Estimation Risk and Optimal Portfolio Choice (North-Holland, Amsterdam ).

Campbell, John Y., 1986, Bond and stock returns in a simple exchange model, Quarterly Journal of Economics 101, 785-803. 
Campbell, John Y., and Robert J. Shiller, 1988, The dividend-price ratio and expectations of future dividends and discount factors, Review of Financial Studies 1, 195-228.

Campbell, John Y., and Robert J. Shiller, 1991, Yield spreads and interest rate movements: A bird's eye view, Review of Economic Studies 58, 495-514.

Campbell, John Y., and Samuel Thompson, 2007, Predicting excess stock returns out of sample: Can anything beat the historical average?, forthcoming, Review of Financial Studies.

Campbell, John Y., and Luis M. Viceira, 1999, Consumption and portfolio decisions when expected returns are time-varying, Quarterly Journal of Economics 114, 433-495.

Campbell, John Y., and Motohiro Yogo, 2006, Efficient tests of stock return predictability, Journal of Financial Economics 81, 27-60.

Cavanagh, Christopher L., Graham Elliott, and James H. Stock, 1995, Inference in models with nearly integrated regressors, Econometric Theory 11, 1131-1147.

Cecchetti, S.G., P.S. Lam, and N.C. Mark, 1993, The equity premium and the risk-free rate: Matching the moments, Journal of Monetary Economics 31, 21-46.

Chib, Siddhartha, and Edward Greenberg, 1995, Understanding the Metropolis-Hastings algorithm, American Statistician 49, 327-335.

Cochrane, John H., 1992, Explaining the variance of price-dividend ratios, Review of Financial Studies 5, 243-280.

Cochrane, John H., 1999, Portfolio advice for a multifactor world, Economic Perspectives Federal Reserve Bank of Chicago 23, 59-78.

Cremers, K.J. Martjin, 2002, Stock return predictability: A Bayesian model selection perspective, Review of Financial Studies 15, 1223-1249.

Cvitanic, Jaksa, Ali Lazrak, Lionel Martellini, and Fernando Zapatero, 2006, Dynamic portfolio choice with parameter uncertainty and the economic value of analysts'recommendations, Review of Financial Studies 19, 1113-1156. 
Eliasz, Piotr, 2004, Optimal median unbiased estimation of coefficients on highly persistent regressors, Working paper, Princeton University.

Elliott, Graham, and James H Stock, 1994, Inference in Time Series Regression When the Order of Integration of a Regressor Is Unknown, Econometric Theory 10, 672-700.

Fama, Eugene F., and Kenneth R. French, 1989, Business conditions and expected returns on stocks and bonds, Journal of Financial Economics 25, 23-49.

Fama, Eugene F., and William G. Schwert, 1977, Asset returns and inflation, Journal of Financial Economics 5, 115-146.

Ferson, Wayne E., Sergei Sarkissian, and Timothy T. Simin, 2003, Spurious regressions in financial economics?, Journal of Finance 58, 1393-1413.

Frost, Peter A., and James Savarino, 1988, For better performance constrain portfolio weights, Journal of Portfolio Management 15, 29-34.

Garlappi, Lorenzo, Raman Uppal, and Tan Wang, 2007, Portfolio selection with parameter and model uncertainty: A multi-prior approach, Review of Financial Studies 20, 41-81.

Goetzmann, William N., and Philippe Jorion, 1993, Testing the predictive power of dividend yields, Journal of Finance 48, 663-679.

Goyal, Amit, and Ivo Welch, 2004, A comprehensive look at the empirical performance of equity premium prediction, Working paper, Brown University and Emory University.

Green, Richard C., and Burton Hollifield, 1992, When will mean-variance efficient portfolios be well diversified?, Journal of Finance 47, 1785-1809.

Green, William H., 1997, Econometric Analysis. (Prentice-Hall, Inc. Upper Saddle River, NJ).

Hamilton, J. D., 1994, Time Series Analysis. (Oxford University Press Princeton, NJ).

Handa, Puneet, and Ashish Tiwari, 2006, Does stock market predictability imply improved asset allocation and performance? Evidence from the U.S. stock market (1954-2002), Journal of Business 79, 2423-2468. 
Hodrick, Robert J., 1992, Dividend yields and expected stock rturns: Alternative procedures for inference and measurement, Review of Financial Studies 5, 357-386.

Jagannathan, Ravi, and Tongshu Ma, 2003, Risk reduction in large portfolios: Why imposing the wrong constraint helps, Journal of Finance 58, 1651-1683.

Jeffreys, Harold, 1961, Theory of Probability. (Oxford University Press Clarenden).

Jobson, J. D., and Bob Korkie, 1980, Estimation of Markowitz efficient portfolios, Journal of the American Statistical Association 75, 544-554.

Johannes, Michael, Nicholas Polson, and Jonathan R. Stroud, 2002, Sequential optimal portfolio performance: Market and volatility timing, Working paper, Columbia University, University of Chicago, and University of Pennsylvania.

Jones, Christopher S., and Jay Shanken, 2005, Mutual fund performance with learning across funds, Journal of Financial Economics 78, 507-552.

Jorion, Philippe, 1985, International portfolio diversification with estimation risk, Journal of Business 58, 259-278.

Kandel, Shmuel, and Robert F. Stambaugh, 1991, Asset returns and intertemporal preferences, Journal of Monetary Economics 27, 39-71.

Kandel, Shmuel, and Robert F. Stambaugh, 1996, On the predictability of stock returns: An asset allocation perspective, Journal of Finance 51, 385-424.

Keim, Donald B., and Robert F. Stambaugh, 1986, Predicting returns in the stock and bond markets, Journal of Financial Economics 17, 357-390.

Klein, Roger W., and Vijay S. Bawa, 1976, The effect of estimation risk on optimal portfolio choice, Journal of Financial Economics 3, 215-231.

Kothari, S. P., and Jay Shanken, 1997, Book-to-market, dividend yield, and expected market returns: A time-series analysis, Journal of Financial Economics 44, 169-203.

Lettau, Martin, and Sydney C. Ludvigson, 2001, Consumption, aggregate wealth and expected stock returns, Journal of Finance 56, 815-849. 
Lettau, Martin, and Stijn VanNieuwerburgh, 2006, Reconciling the return predictability evidence, forthcoming, Review of Financial Studies.

Lewellen, Jonathan, 2004, Predicting returns with financial ratios, Journal of Financial Economics 74, 209-235.

MacKinlay, Craig A., 1995, Multifactor models do not explain the CAPM, Journal of Financial Economics 38, 3-28.

Mankiw, N.Gregory, and Matthew D. Shapiro, 1986, Do we reject too often? : Small sample properties of tests of rational expectations models, Economics Letters 20, 139-145.

Mehra, Rajnish, and Edward Prescott, 1985, The equity premium puzzle, Journal of Monetary Economics 15, 145-161.

Nelson, C. R., and M. J. Kim, 1993, Predictable stock returns: The role of small sample bias, Journal of Finance 48, 641-661.

Pastor, Lubos, 2000, Portfolio selection and asset pricing models, The Journal of Finance $55,179-223$.

Pastor, Lubos, and Robert F. Stambaugh, 1999, Costs of equity capital and model mispricing, Journal of Finance 54, 67-121.

Pastor, Lubos, and Robert F. Stambaugh, 2002, Investing in equity mutual funds, Journal of Financial Economics 63, 351-380.

Paye, Bradley S., and Allan Timmermann, 2005, Instability of return prediction models, forthcoming, Journal of Empirical Finance.

Phillips, Peter C.B., 1991, To criticize the critics: An objective Bayesian analysis of stochastic trends, Journal of Applied Econometrics 6, 333-364.

Richardson, Matthew, and James H. Stock, 1989, Drawing inferences from statistics based on multiyear asset returns, Journal of Financial Economics 25, 323-348.

Robbins, Herbert, 1964, The empirical Bayes approach to statistical decision problems, The Annals of Mathematical Statistics 35, 1-20. 
Samuelson, Paul, 1965, Proof that properly anticipated prices fluctuate randomly, Industrial Management Review 6, 41-49.

Samuelson, Paul, 1973, Proof that properly discounted present values of assets vibrate randomly, Bell Journal of Economics and Management Science 4, 369-374.

Sangvinatsos, Antonios, and Jessica A. Wachter, 2005, Does the failure of the expectations hypothesis matter for long-term investors?, Journal of Finance 60, 179-230.

Shanken, Jay A., and Ane Tamayo, 2005, Dividend yield, risk, and mispricing: A Bayesian analysis, Working paper, Emory University and London Business School.

Shiller, Robert J., 1981, Do stock prices move too much to be justified by subsequent changes in dividends?, American Economic Review 71, 421-436.

Sims, Christopher A., and Harald Uhlig, 1991, Understanding unit rooters: A helicopter tour, Econometrica 59, 1591-1599.

Skoulakis, Georgios, 2007, Dynamic portfolio choice with Bayesian learning, Working paper, University of Maryland.

Stambaugh, Robert, 1999, Predictive regressions, Journal of Financial Economics 54, 375421.

Torous, Walter, Rossen Valkanov, and Shu Yan, 2004, On predicting stock returns with nearly integrated explanatory variables, Journal of Business 77, 937-966.

Tu, Jun, and Guofu Zhou, 2007, Incorporating economic objectives into Bayesian priors: Portfolio choice under parameter uncertainty, Working paper, Singapore Management University and Washington University in St. Louis.

Viceira, Luis, 1996, Testing for structural change in the predictability of asset returns, Working paper, Harvard University.

Wang, Zhenyu, 2005, A shrinkage approach to model uncertainty and asset allocation, Review of Financial Studies 18, 673-705. 
Xia, Yihong, 2001, Learning about predictability: The effects of parameter uncertainty on dynamic asset allocation, Journal of Finance 56, 205-246.

Zellner, Arnold, 1996, An introduction to Bayesian inference in econometrics. (John Wiley and Sons, Inc. New York, NY). 
Table 1: Posterior Means: Dividend-Price Ratio

\begin{tabular}{cccccc}
\hline & \multicolumn{5}{c}{$P\left(R^{2}>2 \%\right)$} \\
\cline { 2 - 4 } Parameter & 0 & 0.0005 & 0.075 & 0.999 & Reg. \\
\hline$\beta_{\text {bond }}$ & 0.00 & 0.02 & -0.00 & -0.18 & -0.10 \\
$\beta_{\text {stock }}$ & $(0.00)$ & $(0.22)$ & $(0.43)$ & $(0.72)$ & $(0.73)$ \\
& 0.00 & 0.69 & 1.41 & 1.46 & 2.72 \\
$\theta_{1}$ & $(0.00)$ & $(0.62)$ & $(0.97)$ & $(1.09)$ & $(1.52)$ \\
& 0.997 & 0.993 & 0.988 & 0.989 & 0.976 \\
$E\left[r_{\text {bond }} \mid B, \Sigma\right]$ & $0.002)$ & $(0.006)$ & $(0.009)$ & $(0.010)$ & $(0.015)$ \\
& 0.18 & 0.18 & 0.18 & 0.17 & 0.23 \\
$E\left[r_{\text {stock }} \mid B, \Sigma\right]$ & $(0.27)$ & $(0.30)$ & $(0.34)$ & $(1.07)$ & \\
& 1.16 & 1.17 & 1.17 & 1.17 & 1.09 \\
$E[x \mid B, \Sigma]$ & $(0.29)$ & $(0.24)$ & $(0.28)$ & $(0.72)$ & \\
& -3.49 & -3.50 & -3.50 & -3.50 & -3.72 \\
\hline
\end{tabular}

Notes: Posterior means for the predictive coefficients $\beta$, the autoregressive coefficient on the predictor variable $\theta_{1}$, and unconditional posterior means for returns and the predictor variable. Posterior standard deviations are in parentheses. The assets are the ten-year bond and the stock index; the log dividend-price ratio is the predictor variable. Prior beliefs are indexed by $P\left(R^{2}>2 \%\right)$, the probability that the $R^{2}$ from the predictive regression exceeds $2 \%$. $P\left(R^{2}>2 \%\right)=0$ corresponds to the dogmatic prior; $P\left(R^{2}>2 \%\right)=0.999$ corresponds to the diffuse prior. The last column gives results implied by parameters estimated by ordinary least squares regression. For regression estimates, standard errors are in parentheses. $E[r \mid B, \Sigma]=\alpha+\beta \frac{\theta_{0}}{1-\theta_{1}}$ and denotes the frequentist expectation of excess returns. $E[x \mid B, \Sigma]=\frac{\theta_{0}}{1-\theta_{1}}$ and denotes the frequentist expectation of the predictor variable. Data are quarterly from 1952 to 2004. 
Table 2: Posterior Means: Yield spread

\begin{tabular}{cccccc}
\hline & \multicolumn{4}{c}{$P\left(R^{2}>2 \%\right)$} & \\
\cline { 2 - 5 } Parameter & 0 & 0.0005 & 0.075 & 0.999 & Reg. \\
\hline$\beta_{\text {bond }}$ & 0.00 & 0.20 & 0.46 & 0.81 & 0.80 \\
& $(0.00)$ & $(0.14)$ & $(0.20)$ & $(0.26)$ & $(0.26)$ \\
$\beta_{\text {stock }}$ & 0.00 & 0.22 & 0.51 & 0.89 & 0.89 \\
& $(0.00)$ & $(0.28)$ & $(0.42)$ & $(0.55)$ & $(0.56)$ \\
$\theta_{1}$ & 0.74 & 0.73 & 0.74 & 0.75 & 0.74 \\
$E\left[r_{\text {bond }} \mid B, \Sigma\right]$ & $(0.05)$ & $(0.05)$ & $(0.05)$ & $(0.05)$ & $(0.05)$ \\
& 0.21 & 0.21 & 0.21 & 0.21 & 0.23 \\
$E\left[r_{\text {stock }} \mid B, \Sigma\right]$ & $(0.28)$ & $(0.28)$ & $(0.29)$ & $(0.33)$ & \\
\multirow{2}{*}{$E[x \mid B, \Sigma]$} & 1.67 & 1.67 & 1.67 & 1.67 & 1.69 \\
& $(0.58)$ & $(0.59)$ & $(0.60)$ & $(0.63)$ & \\
& 0.97 & 0.97 & 0.97 & 0.97 & 0.99 \\
\hline
\end{tabular}

Notes: Posterior means for the predictive coefficients $\beta$, the autoregressive coefficient on the predictor variable $\theta_{1}$, and unconditional posterior means for returns and the predictor variable. Posterior standard deviations are in parentheses. The assets are the ten-year bond and the stock index; the yield spread is the predictor variable. Prior beliefs are indexed by $P\left(R^{2}>2 \%\right)$, the probability that the $R^{2}$ from the predictive regression exceeds $2 \%$. $P\left(R^{2}>2 \%\right)=0$ corresponds to the dogmatic prior; $P\left(R^{2}>2 \%\right)=0.999$ corresponds to the diffuse prior. The last column gives results implied by parameters estimated by ordinary least squares regression. For regression estimates, standard errors are in parentheses. $E[r \mid B, \Sigma]=$ $\alpha+\beta \frac{\theta_{0}}{1-\theta_{1}}$ and denotes the frequentist expectation of excess returns. $E[x \mid B, \Sigma]=\frac{\theta_{0}}{1-\theta_{1}}$ and denotes the frequentist expectation of the predictor variable. Data are quarterly from 1952 to 2004 . 
Table 3: Out-of-Sample Results: Dividend-Price Ratio

\begin{tabular}{|c|c|c|c|c|c|}
\hline \multirow[b]{2}{*}{ A } & \multicolumn{4}{|c|}{$P\left(R^{2}>2 \%\right)$} & \multirow[b]{2}{*}{ Reg. } \\
\hline & 0 & 0.0005 & 0.075 & 0.999 & \\
\hline & \multicolumn{5}{|c|}{ Panel A: Certainty Equivalent Returns } \\
\hline 2 & 6.98 & 7.66 & 6.76 & 5.25 & -2.63 \\
\hline 5 & 6.78 & 7.05 & 6.66 & 6.03 & 2.86 \\
\hline \multicolumn{6}{|c|}{ Panel B: Sharpe Ratios } \\
\hline & 0.19 & 0.22 & 0.19 & 0.15 & 0.16 \\
\hline
\end{tabular}

Notes: Certainty equivalent returns (Panel A) and Sharpe ratios (Panel B) when the dividend-price ratio is the predictor variable and the assets are the stock index and the ten-year bond. For each year beginning in 1972, the predictive distribution for returns is computed using all data up to that year. Optimal portfolios are then computed quarterly to maximize a mean-variance utility function with risk aversion parameter $A=2,5$; these are combined with actual returns over the following quarter to create out-of-sample returns on the investment strategy. Certainty equivalent returns (CERs) are defined as $E\left[r_{p}\right]-\frac{A}{2} \operatorname{Var}\left[r_{p}\right]$, where $r_{p}$ is the return on the investment strategy and the mean and variance are computed using the sample. Sharpe ratios are the average excess returns on the investment strategy divided by the standard deviation and do not depend on $A$. Prior beliefs are indexed by $P\left(R^{2}>2 \%\right)$, the probability that the $R^{2}$ from the predictive regression exceeds $2 \%$. $P\left(R^{2}>2 \%\right)=0$ corresponds to the dogmatic prior; $P\left(R^{2}>2 \%\right)=0.999$ corresponds to the diffuse prior. Reg. denotes results obtained from ordinary least squares regression. Data are from 1952 to 2004. CERs are in annualized percentages $(\times 400)$, and Sharpe ratios are annualized $(\times 2)$. 
Table 4: Out-of-Sample Results: Yield Spread

\begin{tabular}{|c|c|c|c|c|c|}
\hline \multirow[b]{2}{*}{$A$} & \multicolumn{4}{|c|}{$P\left(R^{2}>2 \%\right)$} & \multirow[b]{2}{*}{ Reg. } \\
\hline & 0 & 0.0005 & 0.075 & 0.999 & \\
\hline & \multicolumn{5}{|c|}{ Panel A: Certainty Equivalent Returns } \\
\hline 2 & 5.61 & 7.58 & 9.19 & 5.47 & 0.65 \\
\hline 5 & 6.26 & 7.06 & 7.72 & 6.25 & 4.35 \\
\hline \multicolumn{6}{|c|}{ Panel B: Sharpe Ratios } \\
\hline & 0.19 & 0.28 & 0.37 & 0.43 & 0.39 \\
\hline
\end{tabular}

Notes: Certainty equivalent returns (Panel A) and Sharpe ratios (Panel B) when the yield spread is the predictor variable and the assets are the stock index and the ten-year bond. For each year beginning in 1972, the predictive distribution for returns is computed using all data up to that year. Optimal portfolios are then computed quarterly to maximize a meanvariance utility function with risk aversion parameter $A=2,5$; these are combined with actual returns over the following quarter to create out-of-sample returns on the investment strategy. Certainty equivalent returns (CERs) are defined as $E\left[r_{p}\right]-\frac{A}{2} \operatorname{Var}\left[r_{p}\right]$, where $r_{p}$ is the return on the investment strategy and the mean and variance are computed using the sample. Sharpe ratios are the average excess returns on the investment strategy divided by the standard deviation and do not depend on $A$. Prior beliefs are indexed by $P\left(R^{2}>2 \%\right)$, the probability that the $R^{2}$ from the predictive regression exceeds $2 \% . P\left(R^{2}>2 \%\right)=0$ corresponds to the dogmatic prior; $P\left(R^{2}>2 \%\right)=0.999$ corresponds to the diffuse prior. Reg. denotes results obtained from ordinary least squares regression. Data are from 1952 to 2004. CERs are in annualized percentages $(\times 400)$, and Sharpe ratios are annualized $(\times 2)$. 
Table 5: Monte Carlo Results: Dividend-Price Ratio

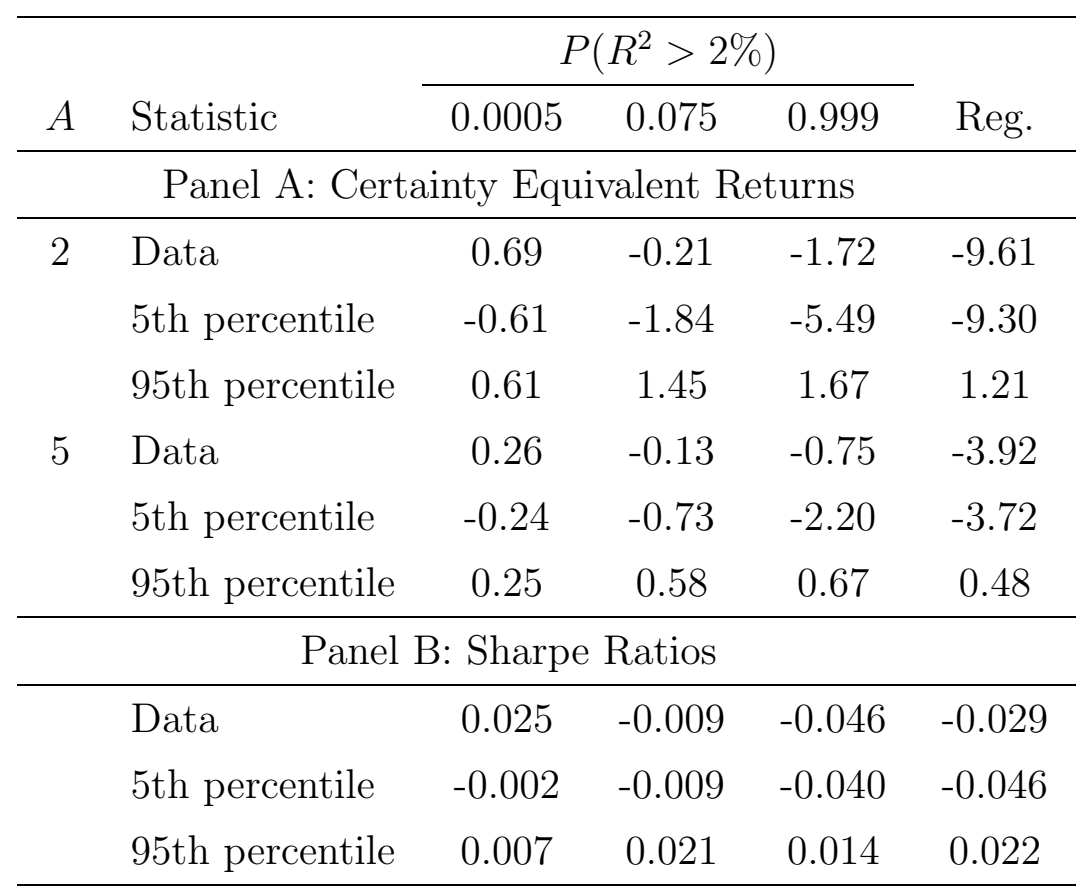

Notes: Differences between the out-of-sample performance of skeptical priors (and of the regression estimates) and that of the dogmatic prior. See Table 3 for the description of the performance measures. "Data" denotes the performance difference in the data. The analysis is repeated in samples of simulated data with no predictability. The table displays the 5 th and 95th percentiles of the performance difference. Prior beliefs are indexed by $P\left(R^{2}>2 \%\right)$, the probability that the $R^{2}$ from the predictive regression exceeds $2 \%$. $P\left(R^{2}>2 \%\right)=0$ corresponds to the dogmatic prior; $P\left(R^{2}>2 \%\right)=0.999$ corresponds to the diffuse prior. Reg. denotes results from ordinary least squares regression. $A$ is the risk-aversion parameter. 
Table 6: Monte Carlo Results: Yield Spread

\begin{tabular}{|c|c|c|c|c|c|}
\hline \multirow[b]{2}{*}{$A$} & \multirow[b]{2}{*}{ Statistic } & \multicolumn{3}{|c|}{$P\left(R^{2}>2 \%\right)$} & \multirow[b]{2}{*}{ Reg. } \\
\hline & & 0.0005 & 0.075 & 0.999 & \\
\hline \multicolumn{6}{|c|}{ Panel A: Certainty Equivalent Returns } \\
\hline \multirow[t]{3}{*}{2} & Data & 1.97 & 3.58 & -0.15 & -4.97 \\
\hline & 5 th percentile & -0.64 & -2.02 & -6.67 & -7.28 \\
\hline & 95th percentile & 0.74 & 1.57 & 1.93 & 1.79 \\
\hline \multirow[t]{3}{*}{5} & Data & 0.80 & 1.46 & -0.01 & -1.90 \\
\hline & 5 th percentile & -0.25 & -0.81 & -2.67 & -2.91 \\
\hline & 95th percentile & 0.30 & 0.63 & 0.77 & 0.72 \\
\hline \multicolumn{6}{|c|}{ Panel B: Sharpe Ratios } \\
\hline & Data & 0.089 & 0.182 & 0.242 & 0.200 \\
\hline & 5th percentile & -0.005 & -0.031 & -0.117 & -0.119 \\
\hline & 95th percentile & 0.001 & -0.009 & -0.055 & -0.055 \\
\hline
\end{tabular}

Notes: Differences between the out-of-sample performance of skeptical priors (and of the regression estimates) and that of the dogmatic prior. See Table 4 for the description of the performance measures. "Data" denotes the performance difference in the data. The analysis is repeated in samples of simulated data with no predictability. The table displays the 5 th and 95th percentiles of the performance difference. Prior beliefs are indexed by $P\left(R^{2}>2 \%\right)$, the probability that the $R^{2}$ from the predictive regression exceeds $2 \% . P\left(R^{2}>2 \%\right)=0$ corresponds to the dogmatic prior; $P\left(R^{2}>2 \%\right)=0.999$ corresponds to the diffuse prior. Reg. denotes results from ordinary least squares regression. $A$ is the risk-aversion parameter. 
Table 7: Subperiod Out-of-Sample Results: Dividend-Price Ratio

\begin{tabular}{|c|c|c|c|c|c|c|}
\hline \multirow[b]{2}{*}{ End Date } & \multirow[b]{2}{*}{$A$} & \multicolumn{4}{|c|}{$P\left(R^{2}>2 \%\right)$} & \multirow[b]{2}{*}{ Reg. } \\
\hline & & 0 & 0.0005 & 0.075 & 0.999 & \\
\hline \multicolumn{7}{|c|}{ Panel A: Certainty Equivalent Returns } \\
\hline 1984 & 2 & 6.64 & 7.99 & 11.05 & 11.42 & -3.14 \\
\hline & 5 & 8.08 & 8.62 & 9.86 & 9.97 & 4.23 \\
\hline 1994 & 2 & 6.35 & 7.27 & 9.34 & 9.03 & 2.60 \\
\hline & 5 & 7.21 & 7.58 & 8.40 & 8.24 & 5.69 \\
\hline \multicolumn{7}{|c|}{ Panel B: Sharpe Ratios } \\
\hline 1984 & & 0.08 & 0.16 & 0.33 & 0.37 & 0.38 \\
\hline 1994 & & 0.10 & 0.15 & 0.28 & 0.29 & 0.34 \\
\hline
\end{tabular}

Notes: Certainty equivalent returns (CERs) and Sharpe ratios are computed as in Table 3 with 1984 and 1994 as end dates rather than 2004. $A$ is the risk aversion parameter. Prior beliefs are indexed by $P\left(R^{2}>2 \%\right)$, the probability that the $R^{2}$ from the predictive regression exceeds $2 \%$. $P\left(R^{2}>2 \%\right)=0$ corresponds to the dogmatic prior; $P\left(R^{2}>2 \%\right)=0.999$ corresponds to the diffuse prior. Reg. denotes out-of-sample results obtained from ordinary least squares regression. CERs are in annualized percentages $(\times 400)$, and Sharpe ratios are annualized $(\times 2)$. 
Table 8: Subperiod Out-of-Sample Results: Yield Spread

\begin{tabular}{|c|c|c|c|c|c|c|}
\hline \multirow[b]{2}{*}{ End Date } & \multirow[b]{2}{*}{$A$} & \multicolumn{4}{|c|}{$P\left(R^{2}>2 \%\right)$} & \multirow[b]{2}{*}{ Reg. } \\
\hline & & 0 & 0.0005 & 0.075 & 0.999 & \\
\hline \multicolumn{7}{|c|}{ Panel A: Certainty Equivalent Returns } \\
\hline 1984 & 2 & 3.11 & 4.74 & 4.83 & -9.24 & -21.91 \\
\hline \multirow{3}{*}{1994} & 5 & 6.67 & 7.36 & 7.44 & 1.92 & -3.07 \\
\hline & 2 & 4.55 & 6.44 & 7.63 & 0.79 & -6.26 \\
\hline & 5 & 6.50 & 7.28 & 7.78 & 5.09 & 2.31 \\
\hline \multicolumn{7}{|c|}{ Panel B: Sharpe Ratios } \\
\hline 1984 & & 0.01 & 0.09 & 0.20 & 0.32 & 0.27 \\
\hline 1994 & & 0.08 & 0.18 & 0.29 & 0.38 & 0.34 \\
\hline
\end{tabular}

Notes: Certainty equivalent returns (CERs) and Sharpe ratios are computed as in Table 4 with 1984 and 1994 as end dates rather than 2004. $A$ is the risk aversion parameter. Prior beliefs are indexed by $P\left(R^{2}>2 \%\right)$, the probability that the $R^{2}$ from the predictive regression exceeds $2 \% . P\left(R^{2}>2 \%\right)=0$ corresponds to the dogmatic prior; $P\left(R^{2}>2 \%\right)=0.999$ corresponds to the diffuse prior. Reg. denotes results from ordinary least squares regression. CERs are in annualized percentages $(\times 400)$, and Sharpe ratios are annualized $(\times 2)$. 
Figure 1: Prior on the $R^{2}$ of the Predictive Regression

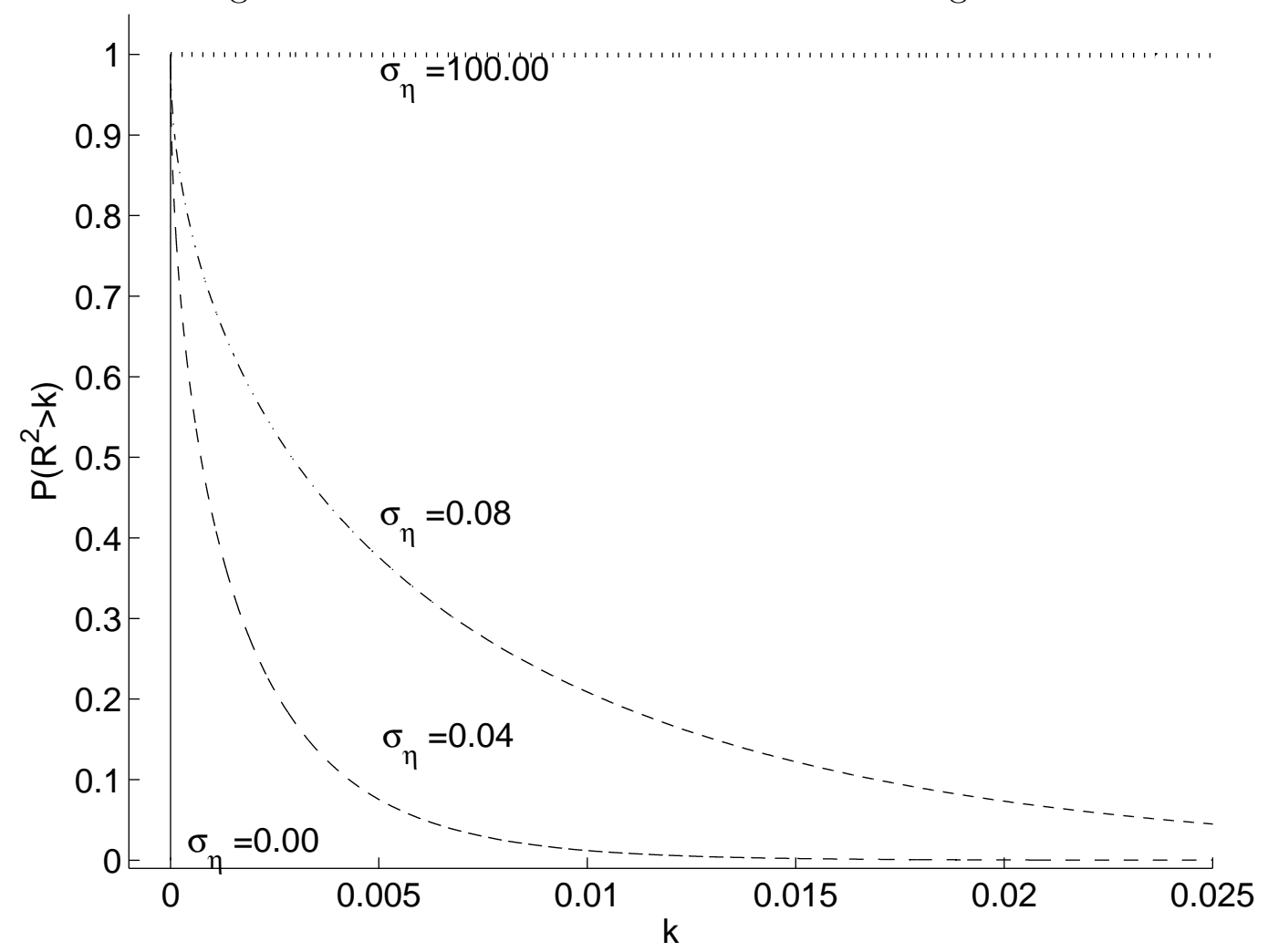

Notes: The prior probability that the $R^{2}$ exceeds a value $k$, for $k$ ranging from 0 to 0.025 implied by skeptical Jeffreys priors. Prior beliefs are indexed by $\sigma_{\eta}$, the prior standard deviation of the normalized coefficient on the predictor variable. The dogmatic prior is given by $\sigma_{\eta}=0$; the diffuse prior by $\sigma_{\eta}=\infty$. Intermediate priors express some skepticism over return predictability. 
Figure 2: Posterior Distribution on the Maximum $R^{2}$ when the Dividend-Price Ratio Predicts Returns
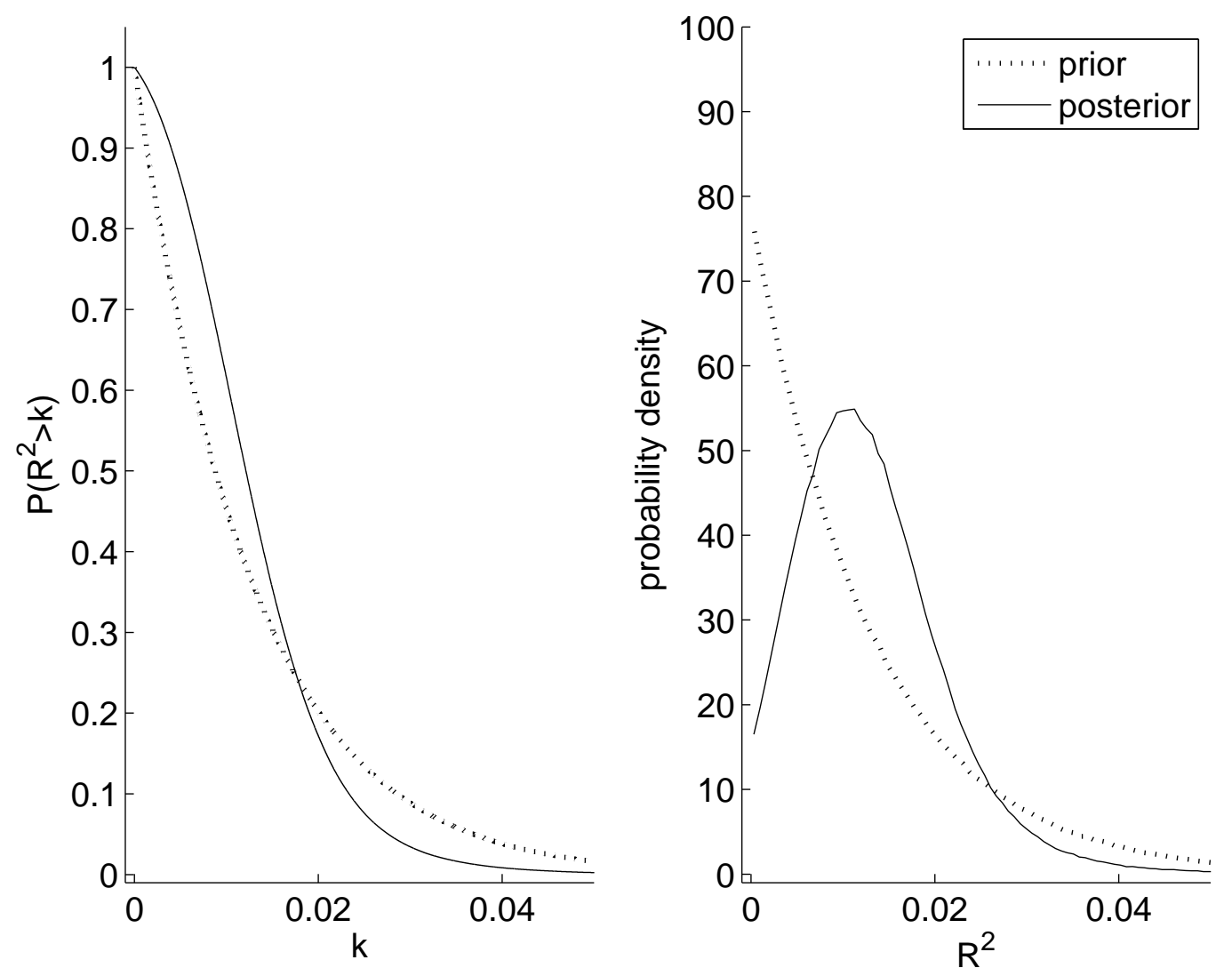

Notes: The left panel shows the prior and the posterior probability that the maximum $R^{2}$ over two assets exceeds a value $k$. The right panel shows the probability density function for the prior and the posterior of the maximum $R^{2}$. The prior volatility of normalized $\beta$, $\sigma_{\eta}$, is set equal to 0.08 . The assets are a stock index and a long-term bond. The predictor variable is the dividend-price ratio and the data are quarterly from 1952 to 2004. 
Figure 3: Posterior Distribution on the Maximum $R^{2}$ when the Yield Spread Predicts Returns
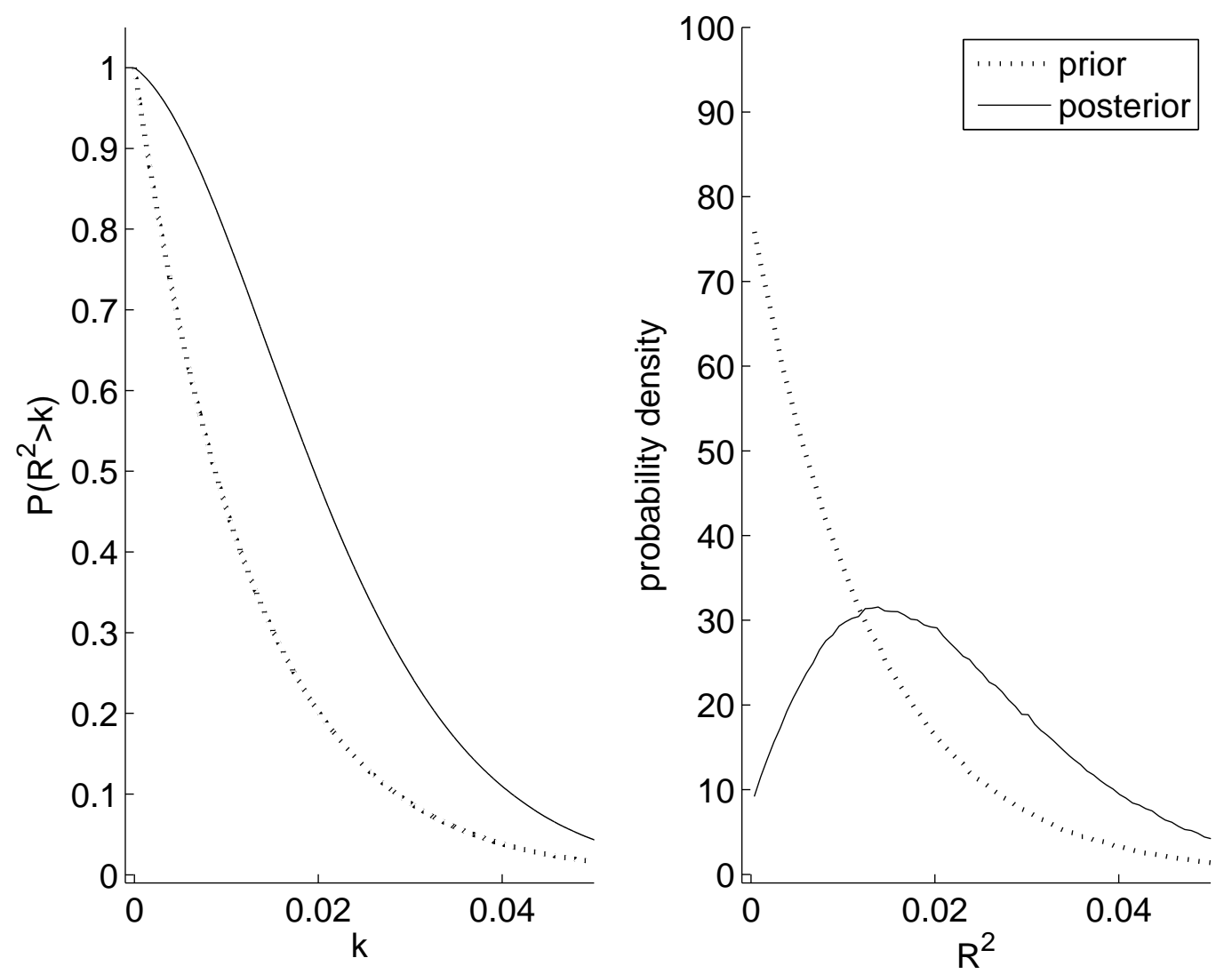

Notes: The left panel shows the prior and the posterior probability that the maximum $R^{2}$ over two assets exceeds a value $k$. The right panel shows the probability density function for the prior and the posterior of the maximum $R^{2}$. The prior volatility of normalized $\beta$, $\sigma_{\eta}$, is set equal to 0.08 . The assets are a stock index and a long-term bond. The predictor variable is the yield spread and the data are quarterly from 1952 to 2004. 
Figure 4: Conditional Expected Returns and Holdings when the Dividend-Price Ratio Predicts Returns

Bond
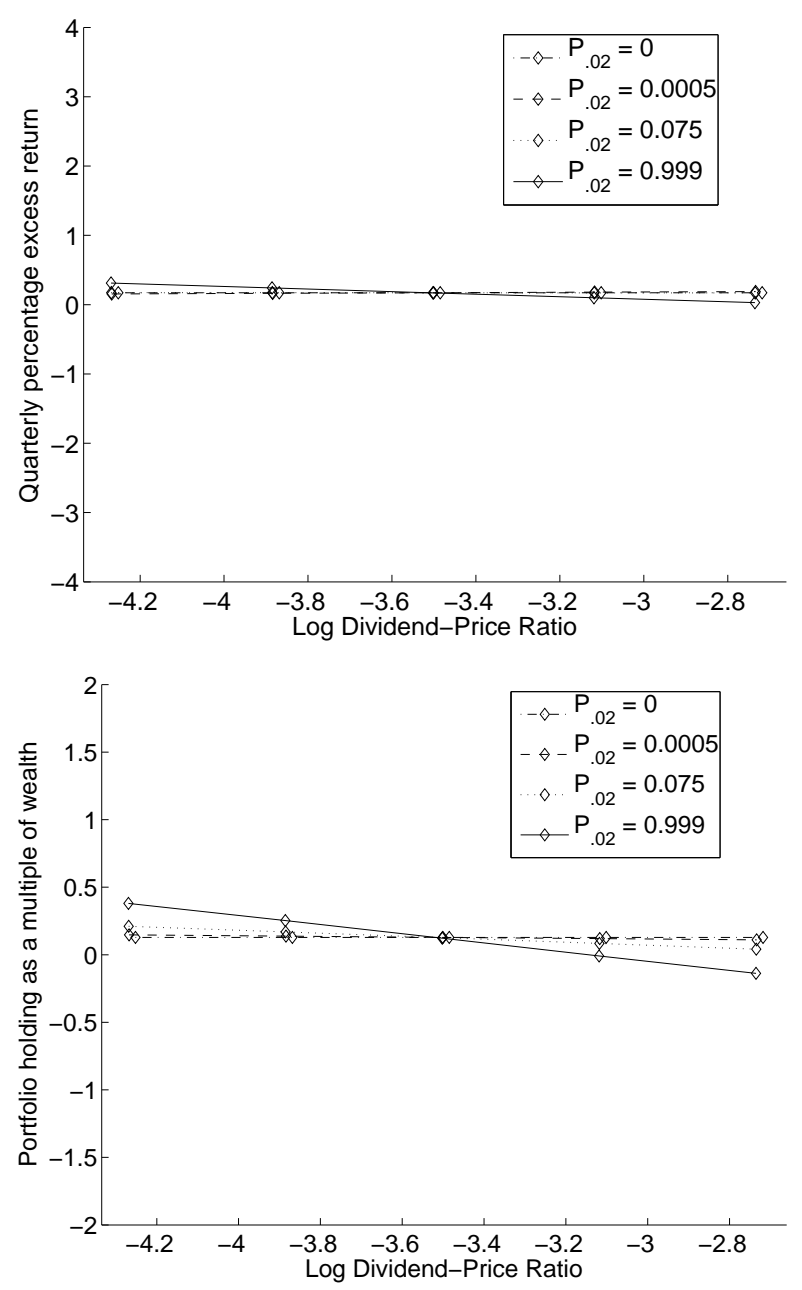

Stock
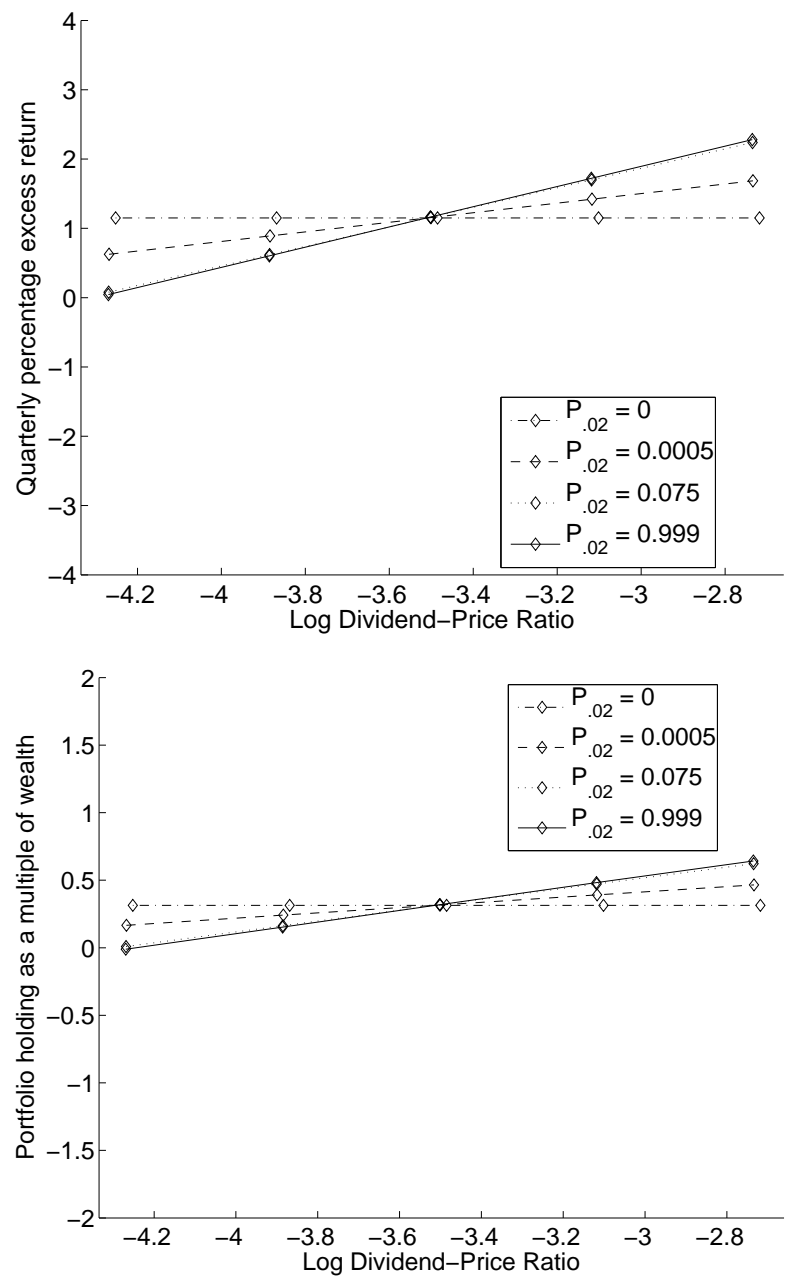

Notes: Conditional expected returns (top two plots) and portfolio holdings (bottom two plots) as functions of the log dividend-price ratio. Conditional expected returns are calculated using the predictive distribution. Given the predictive distribution, portfolios maximize mean-variance utility for risk aversion parameter $A=5$. Assets are a stock index, a long-term bond, and the riskfree asset (not shown). Prior beliefs are indexed by $P_{.02}$, the probability that the $R^{2}$ from the predictive regression exceeds $2 \%$. $P_{.02}=0$ corresponds to the dogmatic prior; $P_{.02}=0.999$ corresponds to the diffuse prior. Diamonds correspond to the sample mean and plus and minus one and two sample standard deviations of the predictor variable. Data are quarterly from 1952 to 2004. 
Figure 5: Conditional Expected Returns and Holdings when the Yield Spread Predicts Returns
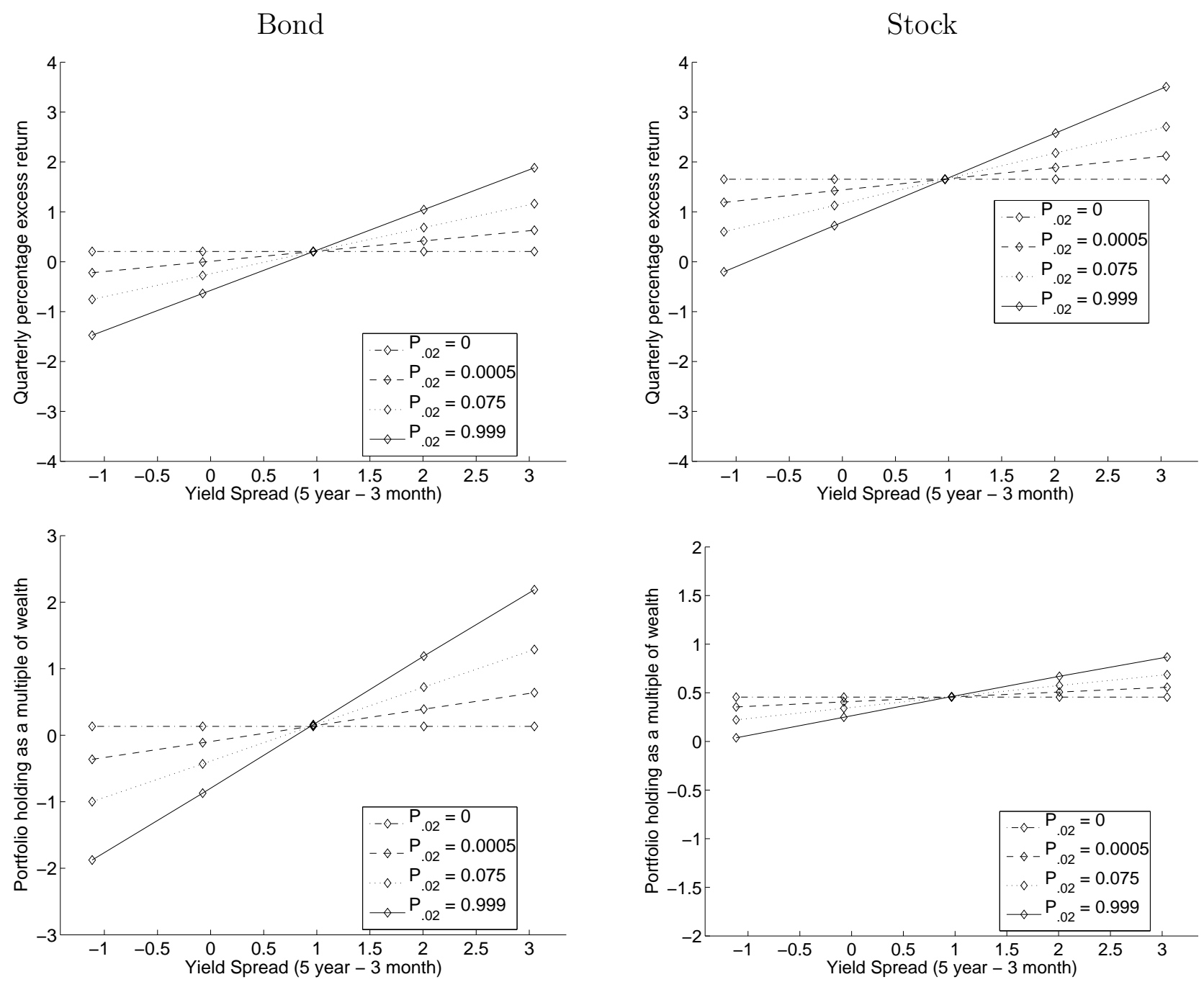

Notes: Conditional expected returns (top two plots) and portfolio holdings (bottom two plots) as functions of the yield spread. Conditional expected returns are calculated using the predictive distribution. Given the predictive distribution, portfolios maximize meanvariance utility for risk aversion parameter $A=5$. Assets are a stock index, a long-term bond, and the riskfree asset (not shown). Prior beliefs are indexed by $P_{.02}$, the probability that the $R^{2}$ from the predictive regression exceeds $2 \%$. $P_{.02}=0$ corresponds to the dogmatic prior; $P_{.02}=0.999$ corresponds to the diffuse prior. Diamonds correspond to the sample mean and plus and minus one and two sample standard deviations of the predictor variable. Data are quarterly from 1952 to 2004. 
Figure 6: Time Series of the Dividend-Price Ratio and Portfolio Holdings for the Diffuse Prior

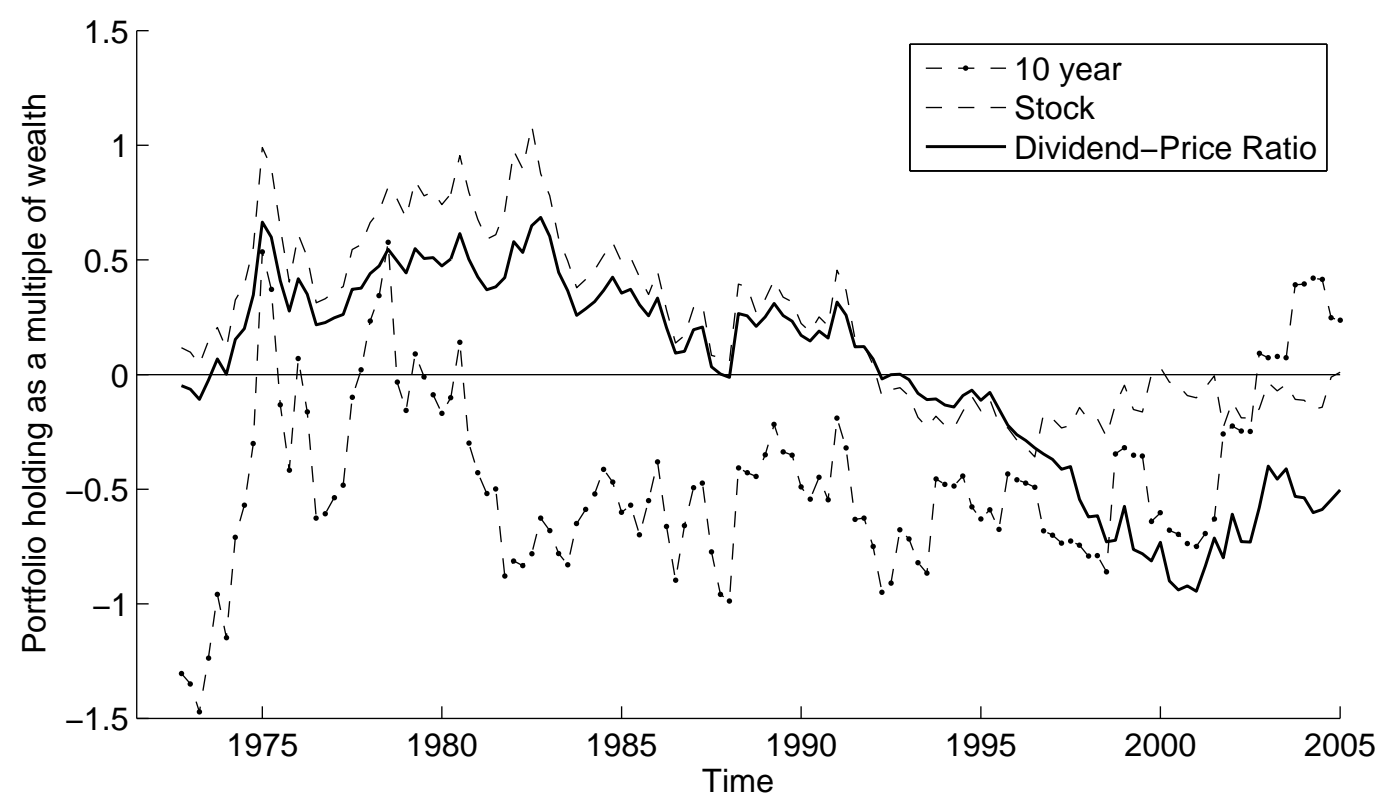

Notes: Time series of optimal portfolio holdings in the long-term (10-year) bond and in the stock index and the de-meaned log dividend-price ratio. Not shown are holdings in the riskfree asset. For each year beginning in 1972, the predictive distribution for returns is computed using the data up to that year assuming that the dividend-price ratio is the predictor variable. Given the predictive distribution, the portfolio maximizes mean-variance utility for risk aversion parameter $A=5$. Data are from 1952 to 2004 . 
Figure 7: Time Series of Posterior Means and Stock Holdings when the Dividend-Price Ratio Predicts Returns

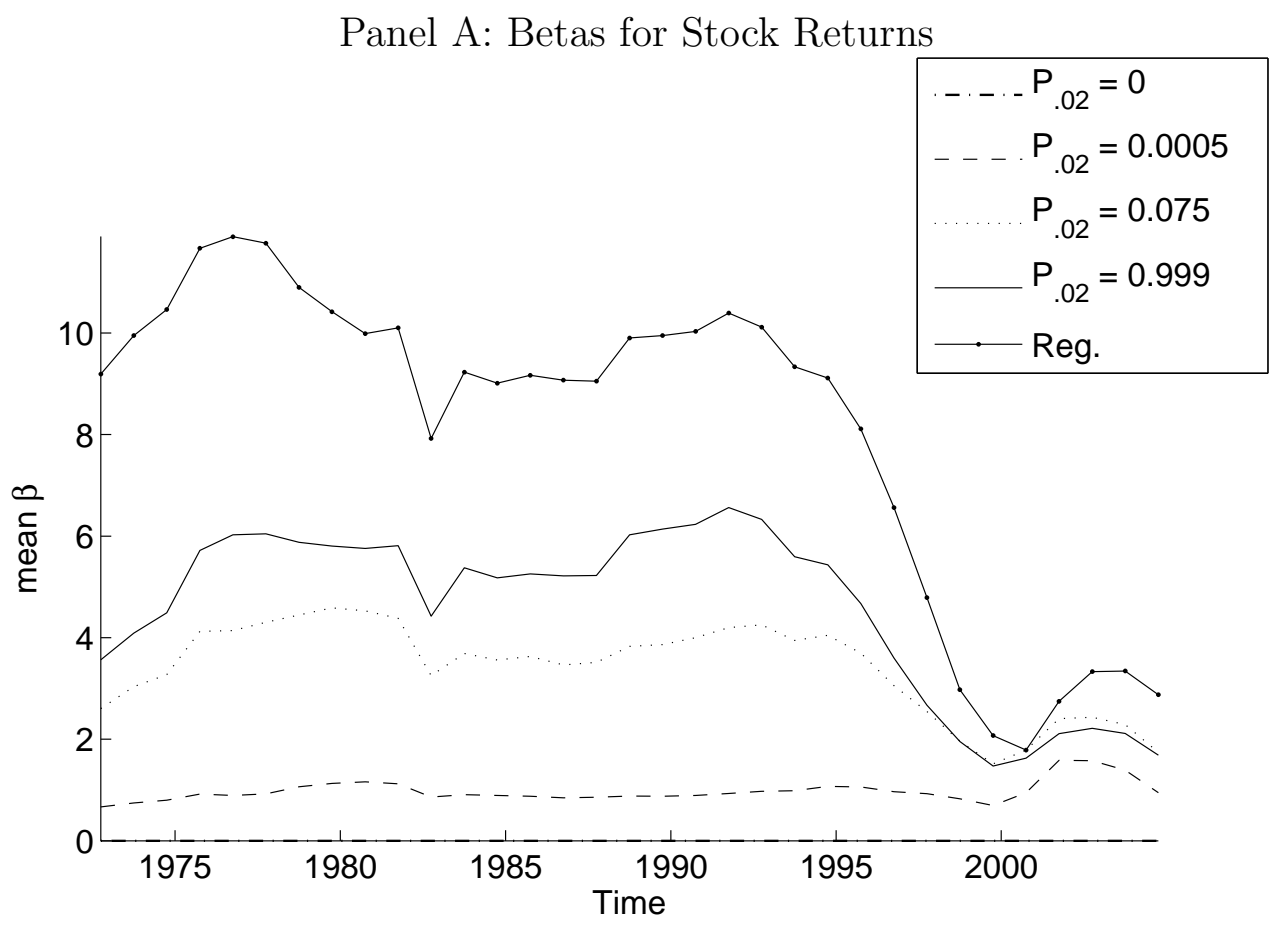

Panel B: Stock Holdings

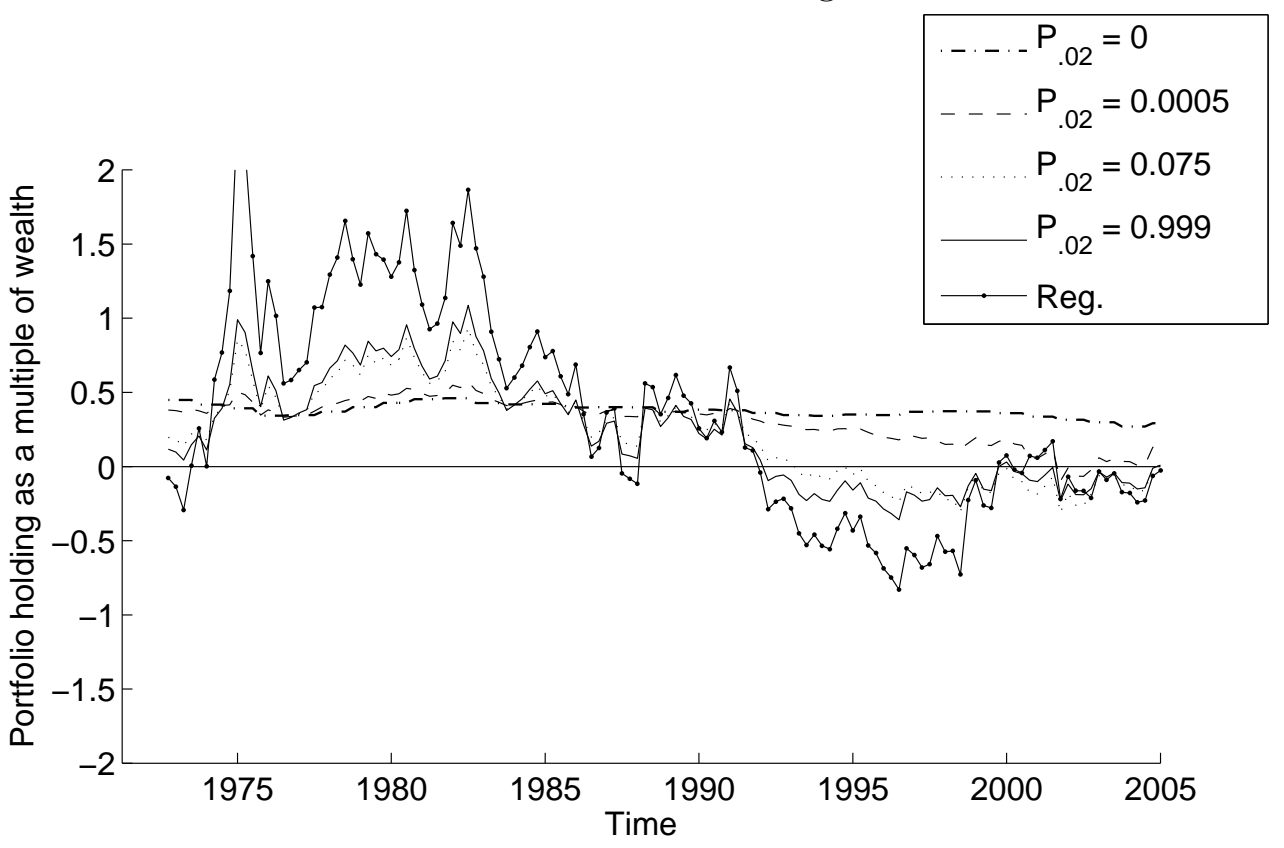

Notes: Panel A shows the posterior mean of $\beta$ (the coefficient on the predictor variable for stock returns); Panel B shows the portfolio holdings in the stock index. For each year beginning in 1972, the posterior distribution is computed using the data up to that year. Optimal portfolios for the mean-variance investor are computed quarterly for risk aversion $A=5$. Prior beliefs are indexed by $P_{.02}$, the probability that the $R^{2}$ from the predictive regression exceeds $2 \%$. $P_{.02}=0$ corresponds to the dogmatic prior; $P_{.02}=0.999$ corresponds to the diffuse prior. Also shown are results from ordinary least squares regression (Reg). Data are quarterly from 1952 to 2004. 
Figure 8: Time Series of Posterior Means and Bond Holdings when the Dividend-Price Ratio Predicts Returns

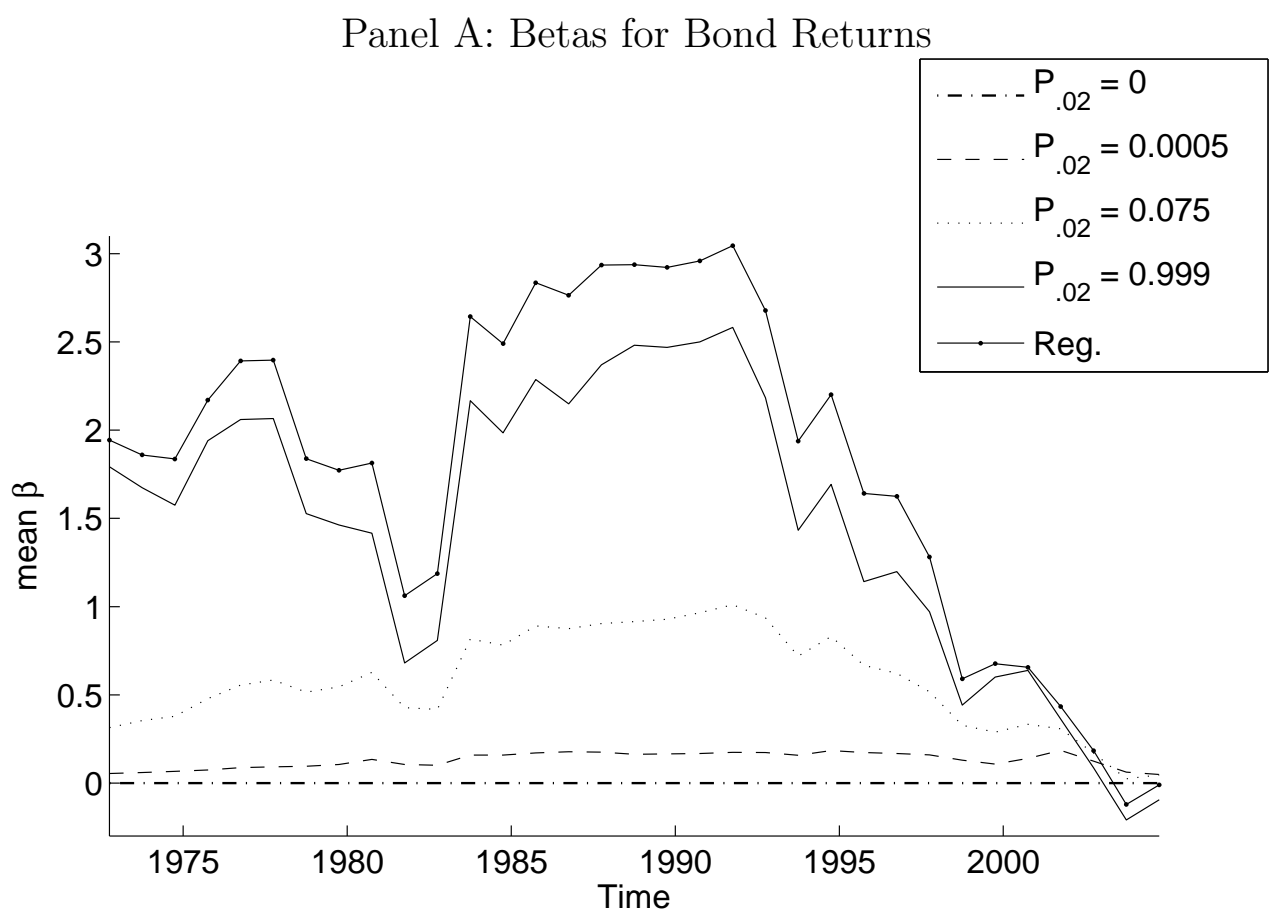

Panel B: Bond Holdings

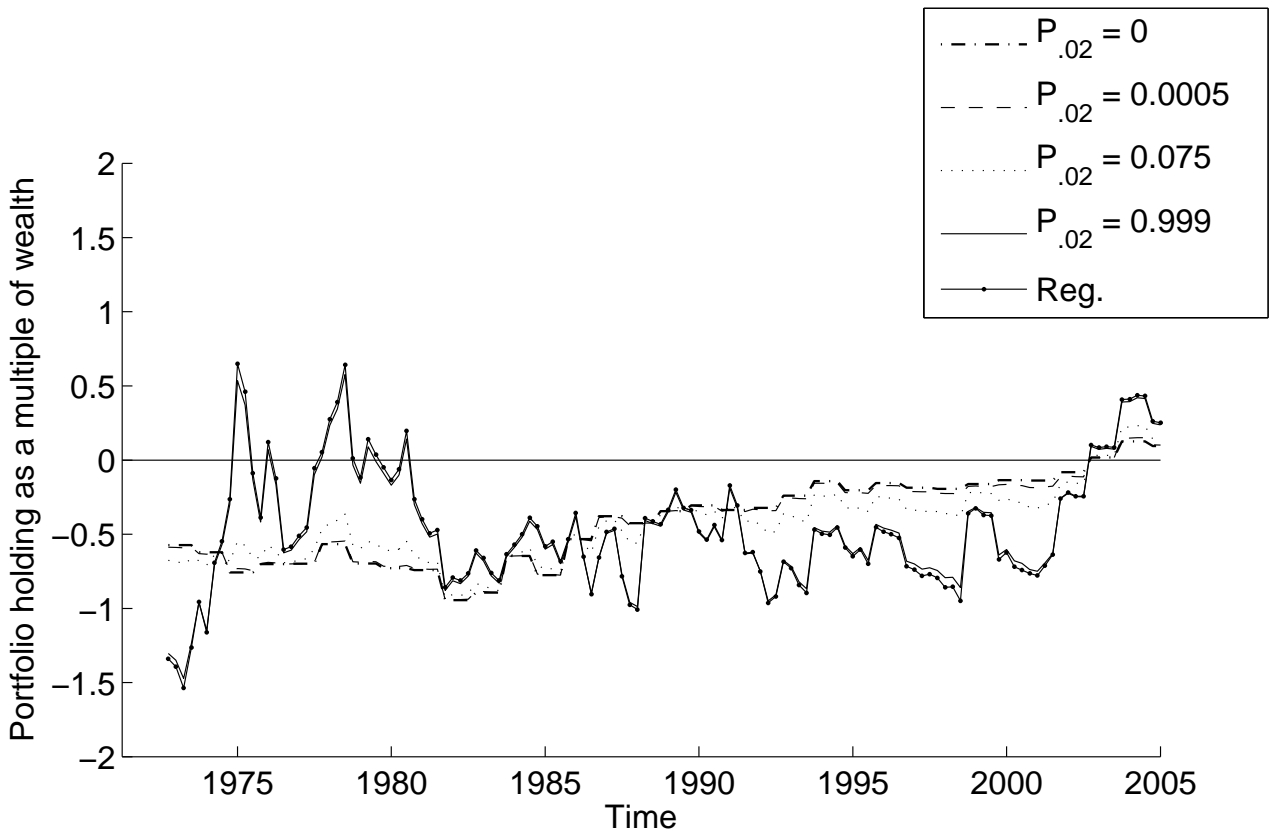

Notes: Panel A shows the posterior mean of $\beta$ (the coefficient on the predictor variable for long-term bond returns); Panel B shows the portfolio holdings in the long-term bond. For each year beginning in 1972, the posterior distribution is computed using the data up to that year. Optimal portfolios for the mean-variance investor are computed quarterly for risk aversion $A=5$. Prior beliefs are indexed by $P_{.02}$, the probability that the $R^{2}$ from the predictive regression exceeds $2 \%$. $P_{.02}=0$ corresponds to the dogmatic prior; $P_{.02}=0.999$ corresponds to the diffuse prior. Also shown are results from ordinary least squares regression (Reg). Data are quarterly from 1952 to 2004. 
Figure 9: Time Series of the Yield Spread and Portfolio Holdings for the Diffuse Prior

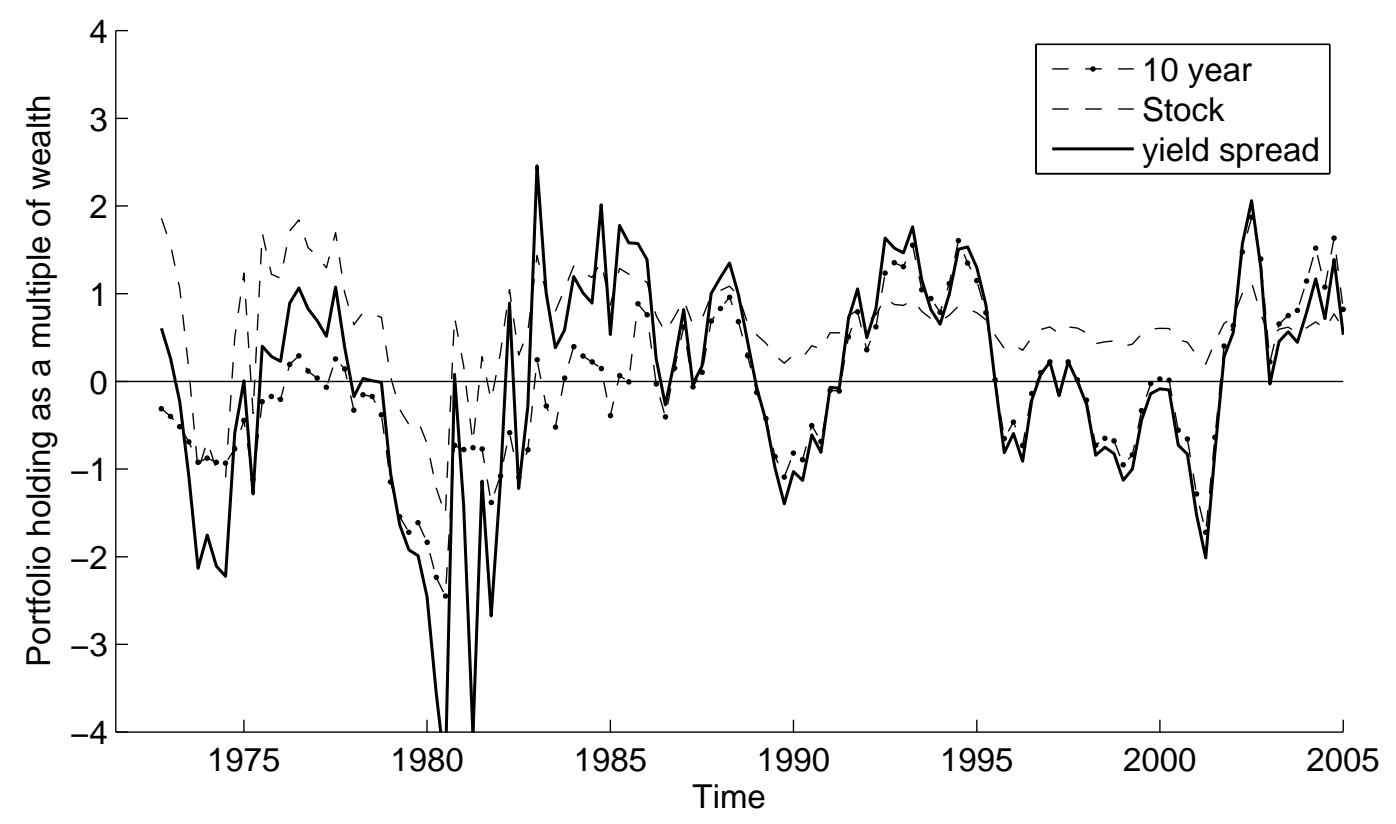

Notes: Time series of optimal portfolio holdings in the long-term (10-year) bond and in the stock index and the de-meaned yield spread. Not shown are holdings in the riskfree asset. For each year beginning in 1972, the predictive distribution for returns is computed using the data up to that year assuming that the yield spread is the predictor variable. Given the predictive distribution, the portfolio maximizes mean-variance utility for risk aversion parameter $A=5$. Data are from 1952 to 2004 . 
Figure 10: Time Series of Posterior Means and Stock Holdings when the Yield Spread Predicts Returns

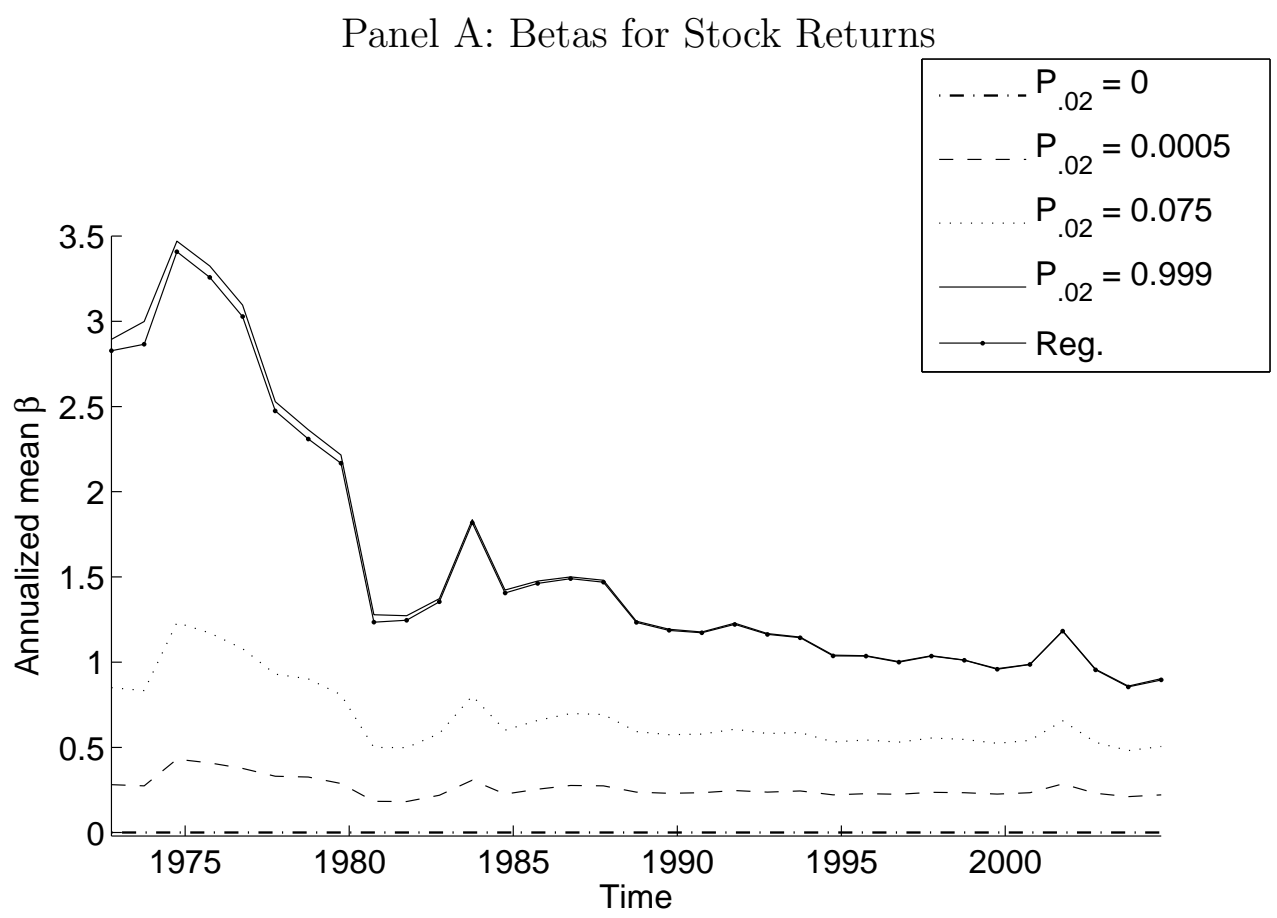

Panel B: Stock Holdings

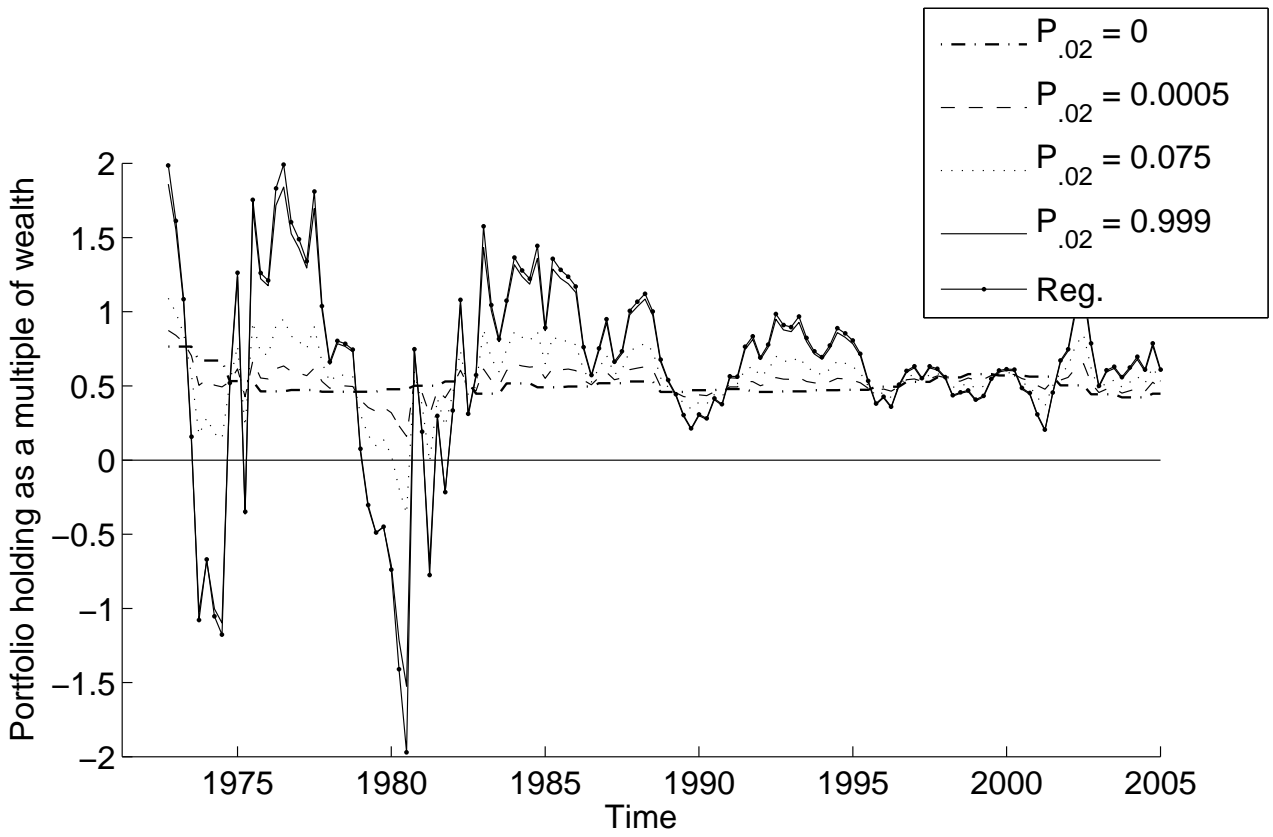

Notes: Panel A shows the posterior mean of $\beta$ (the coefficient on the predictor variable for stock returns); Panel B shows the portfolio holdings in the stock index. For each year beginning in 1972, the posterior distribution is computed using the data up to that year. Optimal portfolios for the mean-variance investor are computed quarterly for risk aversion $A=5$. Prior beliefs are indexed by $P_{.02}$, the probability that the $R^{2}$ from the predictive regression exceeds $2 \%$. $P_{.02}=0$ corresponds to the dogmatic prior; $P_{.02}=0.999$ corresponds to the diffuse prior. Also shown are results from ordinary least squares regression (Reg). Data are quarterly from 1952 to 2004. 
Figure 11: Time Series of Posterior Means and Bond Holdings when the Yield Spread Predicts Returns

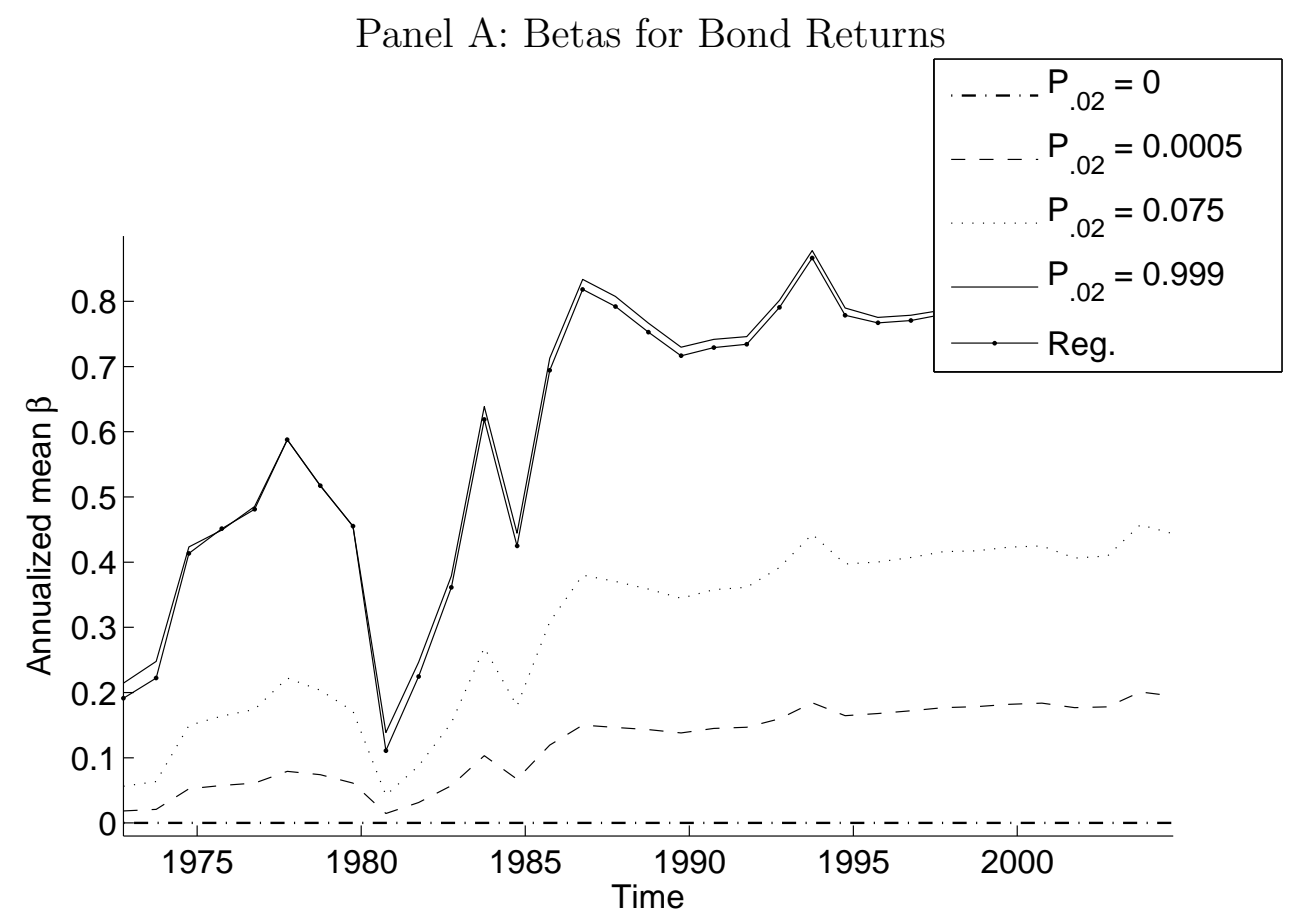

Panel B: Bond Holdings

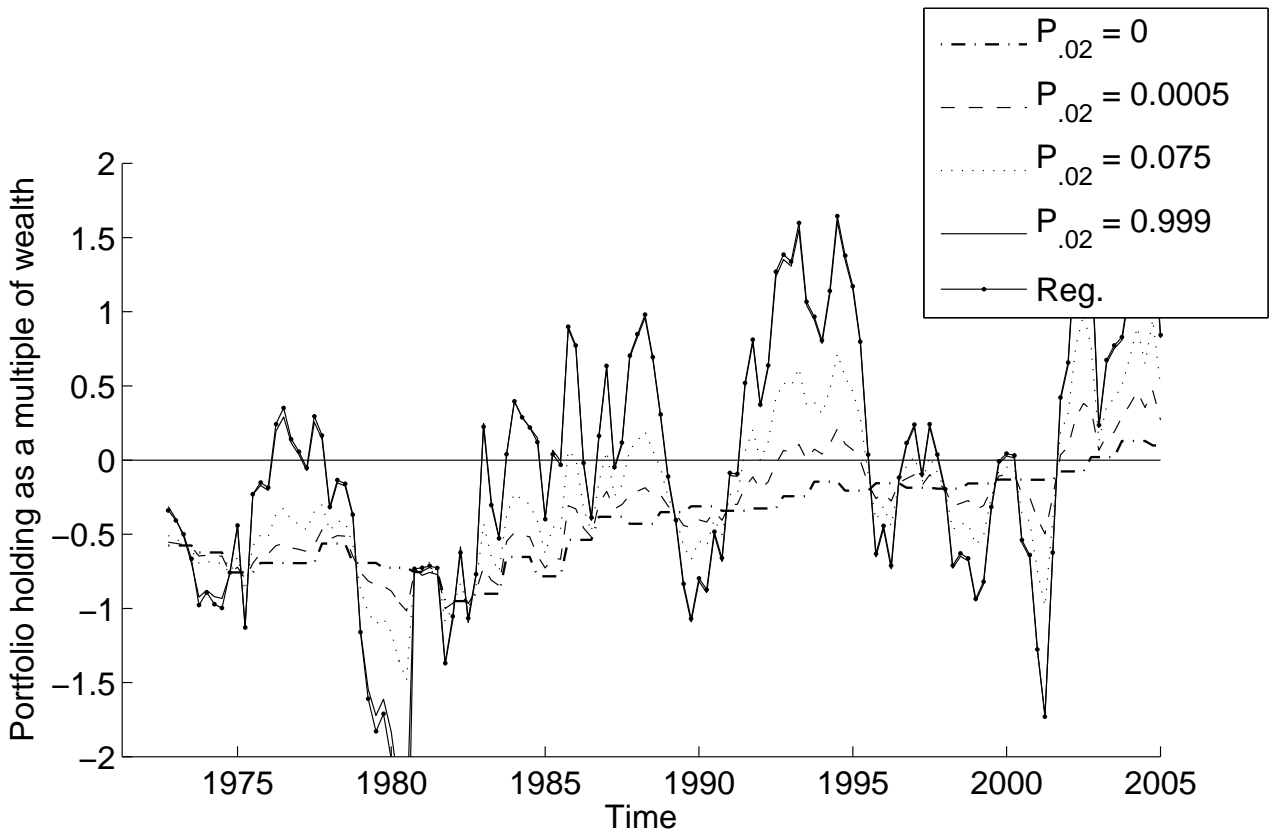

Notes: Panel A shows the posterior mean of $\beta$ (the coefficient on the predictor variable for long-term bond returns); Panel B shows the portfolio holdings in the long-term bond. For each year beginning in 1972, the posterior distribution is computed using the data up to that year. Optimal portfolios for the mean-variance investor are computed quarterly for risk aversion $A=5$. Prior beliefs are indexed by $P_{.02}$, the probability that the $R^{2}$ from the predictive regression exceeds $2 \%$. $P_{.02}=0$ corresponds to the dogmatic prior; $P_{.02}=0.999$ corresponds to the diffuse prior. Also shown are results from ordinary least squares regression (Reg). Data are quarterly from 1952 to 2004. 